\title{
Multiparticle SYM equations of motion and pure spinor BRST blocks
}

\author{
Carlos R. Mafra ${ }^{a}$ Oliver Schlotterer ${ }^{b}$ \\ ${ }^{a}$ DAMTP, University of Cambridge Wilberforce Road, \\ Cambridge, CВ3 0WA, U.K. \\ ${ }^{b}$ Max-Planck-Institut für Gravitationsphysik, \\ Albert-Einstein-Institut, 14476 Potsdam, Germany \\ E-mail: c.r.mafra@damtp.cam.ac.uk, olivers@aei.mpg.de
}

ABSTRACT: In this paper a multiparticle generalization of linearized ten-dimensional super Yang-Mills superfields is proposed. Their equations of motions are shown to take the same form as in the single-particle case, supplemented by contact terms. A recursive construction of these superfields is inspired by the iterated OPEs among massless vertex operators in the pure spinor formalism. An enlarged set of BRST-covariant pure spinor blocks is then defined in a streamlined fashion and combined to multiparticle vertex operators. The latter can be used to universally describe tree-level subdiagrams in the perturbative open and closed superstring, regardless of the loop order. The inherent symmetries of the multiparticle superfields are reproduced by structure constants of the gauge group, hinting a natural appearance of the BCJ-duality between color and kinematics in the fundamentals of super Yang-Mills theory. We present one-loop applications where known scalar cohomology objects are systematically recomputed and a novel vector cohomology particularly relevant to the closed string is constructed for arbitrary multiplicity.

KeYwords: Superstrings and Heterotic Strings, BRST Symmetry

ARXIV EPRINT: 1404.4986 


\section{Contents}

1 Introduction $\quad 2$

2 Review 3

2.1 Ten-dimensional SYM theory 3

2.2 BRST building blocks from vertex operators 4

2.3 Lie symmetries of BRST building blocks 5

2.4 Lie symmetries versus BRST variations $\quad 6$

$\begin{array}{lll}3 & \text { Pure spinor BRST blocks } & 7\end{array}$

3.1 Rank two 7

$\begin{array}{llr}3.2 & \text { Rank three } & 9\end{array}$

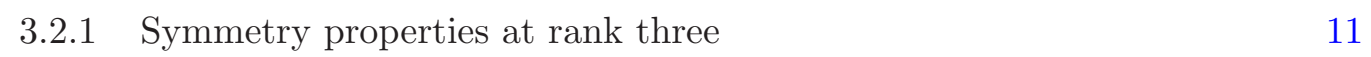

$\begin{array}{lll}3.3 \text { Rank four } & 12\end{array}$

$\begin{array}{lll}\text { 3.3.1 Symmetry properties at rank four } & 14\end{array}$

$\begin{array}{lll}3.4 & \text { Recursive construction at general rank } & 15\end{array}$

4 Berends-Giele currents $\quad 18$

$\begin{array}{lll}4.1 & \text { Symmetries of Berends-Giele currents } 20\end{array}$

5 Application to the one loop cohomology 21

5.1 Tree level SYM amplitudes 21

$\begin{array}{ll}5.2 & \text { Scalar BRST blocks at one-loop } \\ 5.3 & \text { Scalar BRST colom }\end{array}$

$\begin{array}{lll}5.3 & \text { Scalar BRST cohomology at one-loop } & 22\end{array}$

$\begin{array}{lll}5.4 & \text { Vector BRST blocks at one-loop } & 25\end{array}$

$\begin{array}{lll}5.5 & \text { Vector BRST cohomology at one-loop } & 26\end{array}$

6 Conclusion and outlook $\quad 28$

$\begin{array}{ll}\text { A Physics of BRST blocks versus mathematics of cubic graphs } & 29\end{array}$

$\begin{array}{ll}\text { A.1 Iterated bracket notation } & 29\end{array}$

A.2 Diagrammatic representation of BRST blocks and their recursion 30

A.3 Diagrammatic construction of Berends-Giele currents 31

A.4 Different superfield representations versus Lie symmetries 32

B BCJ relations and one-loop scalar cohomology elements 32

$\begin{array}{lll}\text { B.1 A shuffle formula for BCJ relations } & 33\end{array}$

B.2 $\left\langle C_{1 \mid A, B, C}\right\rangle$ from the BCJ shuffle formula 34

$\begin{array}{ll}\text { C The explicit expression for } H_{1234} & 35\end{array}$ 


\section{Introduction}

Many examples have shown that String Theory inspires a deeper understanding of scattering amplitudes in field theories, see e.g. [6-8, 61]. The world-sheet viewpoint on pointparticle interactions offers useful guiding principles through the multitude of Feynman diagrams. For example, tree-level subdiagrams of external particles arise when insertion points of string states on the world-sheet collide. This is captured by the operator product expansion (OPE) among vertex operators.

In this work, we study this mechanism in the context of ten-dimensional super YangMills (SYM) theory. Its superspace description benefits from the use of pure spinors [42-44], and this formulation directly descends from the pure spinor superstring [17]. In previous work, a family of so-called BRST building blocks was identified in the pure spinor formalism [12-14] which encompasses the superfield degrees of freedom of several external particles. These BRST blocks were argued to represent tree-level subdiagrams and led to an elegant and manifestly supersymmetric solution for multileg tree-level amplitudes in SYM theory [12] and the full-fledged open superstring ${ }^{1}[13,14]$. As initially suggested in [45], the driving forces in these constructions were:

(i) The (iterated) OPE of gluon multiplet vertex operators

(ii) The action of the BRST operator on the OPE output to identify the symmetry components in the cohomology

(iii) BRST-invariance of the full amplitude dictates the composition of BRST-covariant tree diagrams

In step (ii), we benefit from the simple form of the BRST action on kinematic degrees of freedom, based on the SYM equations of motion for the superfields [15, 16]. This appears to be special to the pure spinor formalism, at least we are not aware of an analogous implementation in the Ramond-Neveu-Schwarz (RNS) [52-54] or Green-Schwarz (GS) [50, 51] framework.

The tree-level setup of [12-14] only made use of the mixed OPEs between one unintegrated vertex operator $V$ and one integrated version $U$. In recent one- and three-loopcalculations [10, 20,41], on the other hand, it became clear that pieces of the OPE among $U$ vertices had similar covariant BRST properties leading to a simplified description of the amplitudes. In the following, we will complete the list of such BRST-covariant OPE ingredients and introduce multiparticle versions of the integrated vertex operator.

The multiparticle vertex operators are defined in terms of multiparticle superfields of ten-dimensional SYM theory. The latter in turn are constructed recursively where the rule for adding particles is extracted from the OPE among single particle vertex operators. The BRST transformation of these vertex operators is equivalent to equations of motion for the multiparticle superfields, which take the same form as their single-particle counterparts $[15,16]$, but are enriched by contact terms. It points to very fundamental structures

\footnotetext{
${ }^{1}$ See [5] for an indirect derivation of open superstring trees among gluons, based on bosonic gauge invariance and kinematic constraints from the RNS worldsheet prescription.
} 
of SYM theory that these combinations of single-particle fields reproduce the "elementary" equations of motions.

In more mathematical terms $[3,4,24]$, the recursion rule fusing two multiparticle superfields to a larger representative can be viewed as a Lie bracket operation which implements the algebraic structure of tree level graphs. In particular, the aforementioned contact terms present in multiparticle equations of motion directly realize the Lie symmetries of tree subdiagrams. This carries the flavour of a kinematic algebra which might shed further light on the duality between color and kinematics [26] in ten dimensions. ${ }^{2}$ More specifically, the Lie symmetries of multiparticle BRST blocks imply kinematic Jacobi relations within the corresponding tree subdiagrams.

The multiparticle superfields and their BRST properties turn out to guide the construction of BRST-invariant kinematic factors. Together with the tight contraints from zero-mode saturation, this allows to anticipate the structure of scattering amplitudes in both field theory and string theory. As an example, we conclude this paper with an application to one-loop amplitudes of the open and closed (type II) superstring. The pure spinor formulation of the five graviton amplitude in [20] gave an example of how vector contractions between left- and right-moving superfields can be implemented in a BRST-invariant way. The backbone of this superspace construction is a vectorial BRST cohomology element which we recursively extend to higher multiplicity. From the field theory perspective, this amounts to identifying loop momentum dependent parts of the numerators, see [21, 56].

The limit of infinite string tension $\alpha^{\prime} \rightarrow 0$ leads to a worldline realization of the pure spinor setup [1] (see also [47, 48] for the RNS equivalent). It has been shown in [2] that the worldline modifications of the worldsheet vertex operators and their OPEs give rise to the same SYM tree amplitudes as previously obtained from superstring methods [12-14]. Accordingly, it would be interesting to find the worldline equivalent of the present BRST block constructions.

\section{Review}

\subsection{Ten-dimensional SYM theory}

Linearized super-Yang-Mills theory in ten dimensions can be described using the superfields ${ }^{3} A_{\alpha}(x, \theta), A_{m}(x, \theta), W^{\alpha}(x, \theta)$ and $F_{m n}(x, \theta)$ satisfying $[15,16]$

$$
\begin{aligned}
2 D_{(\alpha} A_{\beta)} & =\gamma_{\alpha \beta}^{m} A_{m} & D_{\alpha} A_{m} & =\left(\gamma_{m} W\right)_{\alpha}+k_{m} A_{\alpha} \\
D_{\alpha} F_{m n} & =2 k_{[m}\left(\gamma_{n]} W\right)_{\alpha} & D_{\alpha} W^{\beta} & =\frac{1}{4}\left(\gamma^{m n}\right)_{\alpha}{ }^{\beta} F_{m n} .
\end{aligned}
$$

with gauge transformations $\delta A_{\alpha}=D_{\alpha} \Omega$ and $\delta A_{m}=k_{m} \Omega$ for any superfield $\Omega$. The above equations of motion imply that the superfields $A_{m}, W^{\alpha}$ and $F^{m n}$ can be derived from the

\footnotetext{
${ }^{2}$ See [40],[64] for related work on the kinematic algebra in four and arbitrary dimensions.

${ }^{3}$ It is customary to use a calligraphic letter for the superfield field-strength. However in this paper calligraphic letters will denote the Berends-Giele currents associated to the superfields, see section 4.
} 
spinor superpotential $A_{\alpha}$,

$$
A^{m}=\frac{1}{8}\left(D \gamma^{m} A\right), \quad W^{\alpha}=-\frac{1}{10} \gamma_{m}^{\alpha \beta}\left(k^{m} A_{\beta}-D_{\beta} A^{m}\right), \quad F_{m n}=\frac{1}{8}\left(\gamma_{m n}\right)_{\alpha}{ }^{\beta} D_{\beta} W^{\alpha} .
$$

The notion that the superfield $A_{\alpha}$ is enough to derive the others will be used in the next section to obtain a multiparticle generalization of the above equations of motion.

\subsection{BRST building blocks from vertex operators}

In the pure spinor formalism the massless sector of the open superstring (i.e. the gluon multiplet) is described by the vertex operators

$$
V=\lambda^{\alpha} A_{\alpha}, \quad U=\partial \theta^{\alpha} A_{\alpha}+\Pi^{m} A_{m}+d_{\alpha} W^{\alpha}+\frac{1}{2} N^{m n} F_{m n} .
$$

The superfields $K \in\left\{A_{\alpha}, A_{m}, W^{\alpha}, F_{m n}\right\}$ and the pure spinor ghost $\lambda^{\alpha}$ carry conformal weight zero whereas the worldsheet fields $\left\{\partial \theta^{\alpha}, \Pi^{m}, d_{\alpha}, N^{m n}\right\}$ have conformal weight one. When the superfields are on-shell and the pure spinor constraint $\left(\lambda \gamma^{m} \lambda\right)=0$ is imposed, the vertices satisfy [17]

$$
Q V=0, \quad Q U=\partial V,
$$

where $Q=\lambda^{\alpha} D_{\alpha}$ is the BRST charge. The above fields obey the following OPEs [17, 62],

$$
\begin{aligned}
d_{\alpha}\left(z_{i}\right) K\left(z_{j}\right) & \rightarrow \frac{D_{\alpha} K}{z_{i j}}, & \Pi^{m}\left(z_{i}\right) K\left(z_{j}\right) & \rightarrow-\frac{k^{m} K}{z_{i j}}, \\
d_{\alpha}\left(z_{i}\right) \Pi^{m}\left(z_{j}\right) & \rightarrow \frac{\left(\gamma^{m} \partial \theta\right)_{\alpha}}{z_{i j}} & \Pi^{m}\left(z_{i}\right) \Pi^{n}\left(z_{j}\right) & \rightarrow-\frac{\eta^{m n}}{z_{i j}^{2}}, \\
d_{\alpha}\left(z_{i}\right) \theta^{\beta}\left(z_{j}\right) & \rightarrow \frac{\delta_{\alpha}^{\beta}}{z_{i j}} & d_{\alpha}\left(z_{i}\right) d_{\beta}\left(z_{j}\right) & \rightarrow-\frac{\gamma_{\alpha \beta}^{m} \Pi_{m}}{z_{i j}}, \\
d_{\alpha}\left(z_{i}\right) \partial \theta^{\beta}\left(z_{j}\right) & \rightarrow \frac{\delta_{\alpha}^{\beta}}{z_{i j}^{2}}, & N^{m n}\left(z_{i}\right) \lambda^{\alpha}\left(z_{j}\right) & \rightarrow-\frac{1}{2} \frac{\left(\lambda \gamma^{m n}\right)^{\alpha}}{z_{i j}}
\end{aligned}
$$

and

$$
N^{m n}\left(z_{i}\right) N_{p q}\left(z_{j}\right) \rightarrow \frac{4}{z_{i j}} N_{[p}^{[m} \delta_{q]}^{n]}-\frac{6}{z_{i j}^{2}} \delta_{[p}^{n} \delta_{q]}^{m},
$$

where $z_{i j}=z_{i}-z_{j}$ are worldsheet positions. By $K(x, \theta)$, we collectively denote any superfield containing only zero-modes of $\theta^{\alpha}$ and whose $x$ dependence is entirely given by the plane wave factor ${ }^{4} e^{k \cdot x}$.

Starting with the recursive definition of

$$
\lim _{z_{2} \rightarrow z_{1}} V^{1}\left(z_{1}\right) U^{2}\left(z_{2}\right) \rightarrow \frac{L_{21}}{z_{21}}, \quad \lim _{z_{p} \rightarrow z_{1}} L_{2131 \ldots(p-1) 1}\left(z_{1}\right) U^{p}\left(z_{p}\right) \rightarrow \frac{L_{2131 \ldots(p-1) 1 p 1}}{z_{p 1}},
$$

fermionic ghost-number one BRST building blocks were defined in [12-14] by removal of BRST exact terms,

$$
T_{123 \ldots p}=L_{2131 \ldots p}-Q(\ldots) .
$$

\footnotetext{
${ }^{4}$ To avoid factors of $i$ in the formulae, we define $i k^{m} \equiv k^{m}$.
} 


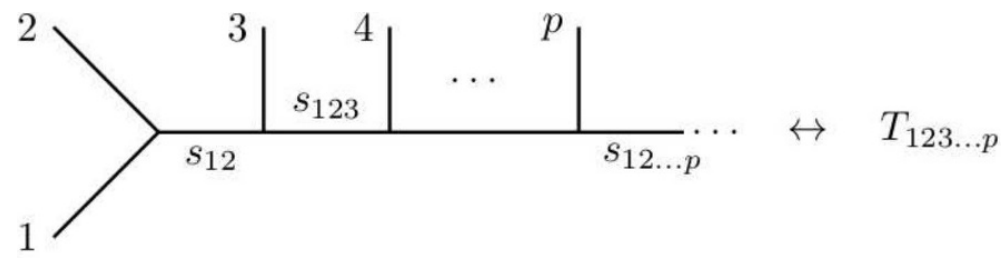

Figure 1. The correspondence of cubic graphs and BRST building blocks.

They transform covariantly under BRST variation, for instance

$$
Q T_{12}=\left(k^{1} \cdot k^{2}\right) T_{1} T_{2}, \quad Q T_{123}=\left(k^{1} \cdot k^{2}\right)\left(T_{1} T_{23}+T_{13} T_{2}\right)+\left(k^{12} \cdot k^{3}\right) T_{12} T_{3}
$$

at rank two and three. More generally,

$$
Q T_{12 \ldots p}=\sum_{j=2}^{p} \sum_{\alpha \in P\left(\beta_{j}\right)}\left(k^{12 \ldots j-1} \cdot k^{j}\right) T_{12 \ldots j-1,\{\alpha\}} T_{j,\left\{\beta_{j} \backslash \alpha\right\}},
$$

where $\beta_{j}=\{j+1, \ldots, p\}$ and $P\left(\beta_{j}\right)$ is the powerset of $\beta_{j}$. Moreover, we identify $T_{i} \equiv V_{i}$ for a single-particle label $i$ and abbreviate multiparticle momenta by $k_{m}^{123 \ldots p} \equiv \sum_{i=1}^{p} k_{m}^{i}$.

\subsection{Lie symmetries of BRST building blocks}

After removal of $Q$ exact terms in (2.8), BRST building blocks $T_{12 \ldots p}$ satisfy all the Lie symmetries $£_{k}$ of tree-level graphs for $2 \leq k \leq p$, where ${ }^{5}$

$$
\begin{array}{rrr}
£_{k=2 n+1}: & T_{12 \ldots n+1[n+2[\ldots[2 n-1[2 n, 2 n+1]] \ldots]]}-T_{2 n+1 \ldots n+2[n+1[\ldots[3[21]] \ldots]]}=0 \\
£_{k=2 n}: & T_{12 \ldots n[n+1[\ldots[2 n-2[2 n-1,2 n]] \ldots]]}+T_{2 n \ldots n+1[n[\ldots[3[21]] \ldots]]}=0 .
\end{array}
$$

Defining the operator $£_{k} \circ$ as the "Lie symmetry generator", the first few examples of the symmetries (2.10) are

$$
\begin{aligned}
& 0=£_{2} \circ T_{12} \equiv T_{12}+T_{21}, \\
& 0=£_{3} \circ T_{123} \equiv T_{123}+T_{231}+T_{312}, \\
& 0=£_{4} \circ T_{1234} \equiv T_{1234}-T_{1243}+T_{3412}-T_{3421} .
\end{aligned}
$$

The symmetries (2.10) have been denoted "Lie" because a contraction of Lie algebra structure constants satisfies the same symmetries [10], ${ }^{6}$

$$
T_{1234 \ldots p} \leftrightarrow f^{12 a_{2}} f^{a_{2} 3 a_{3}} f^{a_{3} 4 a_{4}} \ldots f^{a_{p-1} p a_{p}}
$$

and therefore the building blocks have the correct behavior to describe the kinematic numerators of cubic graphs, see figure 1 .

\footnotetext{
${ }^{5}$ Throughout this work, antisymmetrization over $N$ labels associated with external particles (as in (2.10)) does not contain an overall $1 / N$ !. However, antisymmetrized Lorentz indices $m, n, p, \ldots$ are presented in the convention $A_{[m n]}=\frac{1}{2}\left(A_{m n}-A_{n m}\right)$.

${ }^{6}$ Under the Dynkin bracketing operation, the building blocks satisfy $T_{[[\ldots[[1,2], 3], \ldots], p]}=p T_{123 \ldots p}$ and therefore they belong to $\operatorname{Lie}(p)$. See e.g. Proposition 13.2.3 of [24].
} 


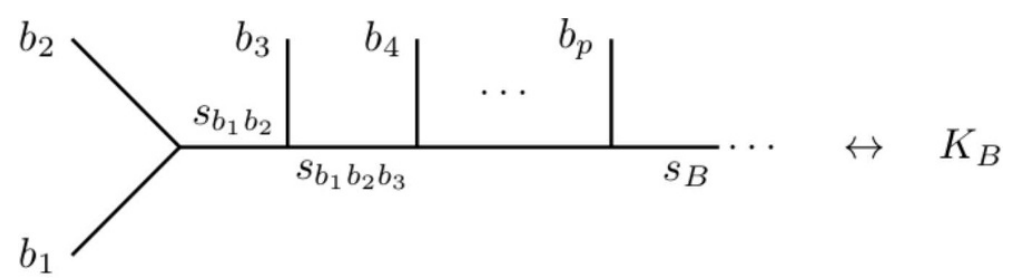

Figure 2. Four superfield realizations $K_{B} \in\left\{A_{\alpha}^{B}, A_{m}^{B}, W_{B}^{\alpha}, F_{m n}^{B}\right\}$ of cubic tree graphs $B=$ $b_{1} b_{2} \ldots b_{p}$. This generalizes the mapping in figure 1 from previous work [12-14] where only one representative $T_{B}$ at ghost number one was given.

\subsection{Lie symmetries versus BRST variations}

It is crucial to notice the interplay between the BRST variations (2.9) and the Lie symmetries (2.10) of cubic tree level subdiagrams: at rank two and three, we have

$$
Q\left(T_{12}+T_{21}\right)=0, \quad Q\left(T_{123}+T_{213}\right)=Q\left(T_{123}+T_{231}+T_{312}\right)=0
$$

and the BRST variation (2.9) always has the precise form to make the sums in (2.10) BRST closed. This closure even holds before the redefinitions (2.8) are performed, e.g. $Q\left(L_{12}+L_{21}\right)=0$ for the direct outcome of the OPE (2.7). Any such BRST closed combination is also BRST exact since its conformal weight $\sim k_{12 \ldots p}^{2}$ is different from zero (unless $p=1){ }^{7}$ As detailed in [12-14], this implies that BRST exact terms (such as $\left.\left.Q\left(A_{1} \cdot A_{2}\right)=L_{21}+L_{12}\right)\right)$ can be subtracted in the definition of $T_{12 \ldots p}$ given in (2.8). Therefore the Lie symmetries obeyed by $T_{12 \ldots p}$ are a consequence of the underlying BRST cohomology nature of the pure spinor superspace expressions which will ultimately describe the scattering amplitudes.

However, it was a matter of trial and error to find the BRST- "ancestors" of $Q$-closed $L_{21 \ldots p 1}$ combinations, such as $\left(A_{1} \cdot A_{2}\right)$ in the rank-two example and more lengthy expression at rank $\leq 5$ given in $[13,14]$. In the following section, we develop a constructive method to generate these BRST completions in (2.8) without any guesswork. Moreover, our current approach based on integrated vertex operators $U_{i}$ delays the need for redefinitions (2.8) to rank three; all the rank-two BRST blocks will automatically be antisymmetric since they follow from the simple pole of the OPE between two integrated vertices.

The BRST building blocks play a key role in the recursive BRST cohomology method to compute SYM tree-level amplitudes $[12,45]$ and in obtaining a manifestly local representation of BCJ-satisfying [26] tree-level numerators [23]. However, their explicit superspace expressions in $[13,14]$ following from the more and more cumbersome OPE computations (2.7) become lengthy for higher rank and lack an organizing principle. We will describe a recursive method in the next section to find compact representations and to completely bypass the CFT calculations beyond rank two.

\footnotetext{
${ }^{7}$ Recall that in a topological conformal field theory $Q b_{0}=L_{0}$ implies that if $Q \phi_{h}=0$ and $L_{0} \phi_{h}=h \phi_{h}$, then $\phi_{h}=(1 / h) Q\left(b_{0} \phi_{h}\right)$ for $h \neq 0$. See e.g. [35].
} 


\section{$3 \quad$ Pure spinor BRST blocks}

In this section we will show how to recursively define multiparticle superfields $A_{\alpha}^{B}(x, \theta)$, $A_{B}^{m}(x, \theta), W_{B}^{\alpha}(x, \theta)$ and $F_{B}^{m n}(x, \theta)$. As we will see, the recursion is driven by the OPE among two single-particle vertex operators and a system of multiparticle SYM equations of motion which generalize the standard description of (2.1). Throughout this paper, upper case letters from the beginning of the Latin alphabet will represent multiparticle labels, e.g. $B=b_{1} b_{2} \ldots b_{p}$ at rank $p \equiv|B|$. In particular, whenever they are attached to a multiparticle superfield $K_{B} \in\left\{A_{\alpha}^{B}, A_{m}^{B}, W_{B}^{\alpha}, F_{m n}^{B}\right\}$ (without any hats or primes), the $B=b_{1} b_{2} \ldots b_{p}$ carry the same Lie symmetries (2.10),

$$
\begin{array}{rrr}
£_{k=2 n+1}: & K_{12 \ldots n+1[n+2[\ldots[2 n-1[2 n, 2 n+1]] \ldots]]}-K_{2 n+1 \ldots n+2[n+1[\ldots[3[21]] \ldots]]}=0 \\
£_{k=2 n}: & K_{12 \ldots n[n+1[\ldots[2 n-2[2 n-1,2 n]] \ldots]]}+K_{2 n \ldots n+1[n[\ldots[3[21]] \ldots]]}=0 .
\end{array}
$$

The superfields $\left\{A_{\alpha}^{B}, A_{m}^{B}, W_{B}^{\alpha}, F_{m n}^{B}\right\}$ of multiplicity $p \equiv|B|$ satisfying all the Lie symmetries $£_{k}$ for $k \leq p$ will be referred to as BRST blocks. ${ }^{8}$ Given the symmetry matching relation

$$
K_{1234 \ldots p} \leftrightarrow f^{12 a_{2}} f^{a_{2} 3 a_{3}} f^{a_{3} 4 a_{4}} \ldots f^{a_{p-1} p a_{p}}
$$

with color factors, the BRST blocks reproduce symmetry properties of Lie algebraic structure constants. The BCJ compatibility of the explicit tree-level numerators in [23] are based on $\lambda^{\alpha} A_{\alpha}^{B}$ satisfying this symmetry matching. As described in the mathematics literature $[3,24]$, the associated cubic graphs shown in figure 2 (planar binary trees in mathematical jargon) can be mapped to iterated brackets and thereby give rise to a general construction of a Lie algebra basis. More details are given in appendix A.

The BRST variation of the multiparticle unintegrated vertex operator defined by $V^{B} \equiv$ $\lambda^{\alpha} A_{\alpha}^{B}$ will be shown to have the same functional form as the BRST variation (2.9) of $T_{B}$, thereby constituting a new representation of such objects. BRST-invariants built from $T_{B}$ do not change under a global redefinition $T_{B} \rightarrow V_{B}$, hence the representations are equivalent. From now on, $T_{B}$ from [12-14] will not be used anymore and the new representation $V_{B}$ will take its place because it follows from simpler principles.

\subsection{Rank two}

The way towards multiparticle BRST blocks is suggested by the OPE between two integrated vertex operators. This is the largest and only CFT computation relevant for this work and has been firstly performed in [11],

$$
\begin{aligned}
U^{1}\left(z_{1}\right) U^{2}\left(z_{2}\right) \rightarrow & z_{12}^{-k^{1} \cdot k^{2}-1}\left(\partial \theta^{\alpha}\left[\left(k^{1} \cdot A_{2}\right) A_{\alpha}^{1}-\left(k^{2} \cdot A_{1}\right) A_{\alpha}^{2}+D_{\alpha} A_{\beta}^{2} W_{1}^{\beta}-D_{\alpha} A_{\beta}^{1} W_{2}^{\beta}\right]\right. \\
& +\Pi^{m}\left[\left(k^{1} \cdot A_{2}\right) A_{m}^{1}-\left(k^{2} \cdot A_{1}\right) A_{m}^{2}+k_{m}^{2}\left(A_{2} W_{1}\right)-k_{m}^{1}\left(A_{1} W_{2}\right)-\left(W_{1} \gamma_{m} W_{2}\right)\right] \\
& +d_{\alpha}\left[\left(k^{1} \cdot A_{2}\right) W_{1}^{\alpha}-\left(k^{2} \cdot A_{1}\right) W_{2}^{\alpha}+\frac{1}{4}\left(\gamma^{m n} W_{1}\right)^{\alpha} F_{m n}^{2}-\frac{1}{4}\left(\gamma^{m n} W_{2}\right)^{\alpha} F_{m n}^{1}\right]
\end{aligned}
$$

\footnotetext{
${ }^{8}$ Throughout this paper, we will distinguish BRST building blocks $T_{B}$ as reviewed in section 2 from BRST blocks $K_{B} \in\left\{A_{\alpha}^{B}, A_{m}^{B}, W_{B}^{\alpha}, F_{m n}^{B}\right\}$ to be constructed in this section.
} 


$$
\begin{aligned}
& \left.+\frac{1}{2} N^{m n}\left[\left(k^{1} \cdot A_{2}\right) F_{m n}^{1}-\left(k^{2} \cdot A_{1}\right) F_{m n}^{2}-2 k_{m}^{12}\left(W_{1} \gamma_{n} W_{2}\right)-2 F_{m a}^{2} F_{n}^{3 a}\right]\right) \\
& +\left(1+k^{1} \cdot k^{2}\right) z_{12}^{-k^{1} \cdot k^{2}-2}\left[\left(A_{1} W_{2}\right)+\left(A_{2} W_{1}\right)-\left(A_{1} \cdot A_{2}\right)\right] .
\end{aligned}
$$

Using the relation $\partial K=\partial \theta^{\alpha} D_{\alpha} K+\Pi^{m} k_{m} K$ for superfields $K$ independent on $\partial \theta^{\alpha}$ and $\lambda^{\alpha}$, we can absorb the most singular piece $\sim z_{12}^{-k^{1} \cdot k^{2}-2}$ into total $z_{1}, z_{2}$ derivatives and rewrite

$$
\begin{aligned}
U^{1}\left(z_{1}\right) U^{2}\left(z_{2}\right) \rightarrow & -z_{12}^{-k^{1} \cdot k^{2}-1}\left[\partial \theta^{\alpha} A_{\alpha}^{12}+\Pi^{m} A_{m}^{12}+d_{\alpha} W_{12}^{\alpha}+\frac{1}{2} N^{m n} F_{m n}^{12}\right] \\
& +\partial_{1}\left(z_{12}^{-k^{1} \cdot k^{2}-1}\left[\frac{1}{2}\left(A_{1} \cdot A_{2}\right)-\left(A_{1} W_{2}\right)\right]\right) \\
& -\partial_{2}\left(z_{12}^{-k^{1} \cdot k^{2}-1}\left[\frac{1}{2}\left(A_{1} \cdot A_{2}\right)-\left(A_{2} W_{1}\right)\right]\right)
\end{aligned}
$$

where

$$
\begin{aligned}
A_{\alpha}^{12} & =-\frac{1}{2}\left[A_{\alpha}^{1}\left(k^{1} \cdot A^{2}\right)+A_{m}^{1}\left(\gamma^{m} W^{2}\right)_{\alpha}-(1 \leftrightarrow 2)\right] \\
A_{m}^{12} & =\frac{1}{2}\left[A_{p}^{1} F_{p m}^{2}-A_{m}^{1}\left(k^{1} \cdot A^{2}\right)+\left(W^{1} \gamma_{m} W^{2}\right)-(1 \leftrightarrow 2)\right] \\
W_{12}^{\alpha} & =\frac{1}{4}\left(\gamma^{m n} W^{2}\right)^{\alpha} F_{m n}^{1}+W_{2}^{\alpha}\left(k^{2} \cdot A^{1}\right)-(1 \leftrightarrow 2) \\
F_{m n}^{12} & =F_{m n}^{2}\left(k^{2} \cdot A^{1}\right)+F_{[m}^{2}{ }^{p} F_{n] p}^{1}+k_{12}^{[m}\left(W_{1} \gamma^{n]} W_{2}\right)-(1 \leftrightarrow 2) \\
& =k_{m}^{12} A_{n}^{12}-k_{n}^{12} A_{m}^{12}-\left(k^{1} \cdot k^{2}\right)\left(A_{m}^{1} A_{n}^{2}-A_{n}^{1} A_{m}^{2}\right) .
\end{aligned}
$$

Note that the last line can be viewed as a multiparticle generalization of the field-strength relation $F_{m n}^{i}=k_{m}^{i} A_{n}-k_{n}^{i} A_{m}$, modified by the contact terms $\left(k^{1} \cdot k^{2}\right)\left(A_{m}^{1} A_{n}^{2}-A_{n}^{1} A_{m}^{2}\right)$.

In the prescription for computing string amplitudes the vertex operators are integrated over the worldsheet so the total derivatives can be dropped ${ }^{9}$ and the composite superfields in (3.5) can be picked up via

$$
\begin{aligned}
U^{12} & =-\oint z_{12}^{k^{1} \cdot k^{2}} U^{1}\left(z_{1}\right) U^{2}\left(z_{2}\right) \\
& =\partial \theta^{\alpha} A_{\alpha}^{12}+\Pi^{m} A_{m}^{12}+d_{\alpha} W_{12}^{\alpha}+\frac{1}{2} N^{m n} F_{m n}^{12} .
\end{aligned}
$$

One can check using (2.1) that the above superfields satisfy

$$
\begin{aligned}
2 D_{(\alpha} A_{\beta)}^{12} & =\gamma_{\alpha \beta}^{m} A_{m}^{12}+\left(k^{1} \cdot k^{2}\right)\left(A_{\alpha}^{1} A_{\beta}^{2}+A_{\beta}^{1} A_{\alpha}^{2}\right) \\
D_{\alpha} A_{m}^{12} & =\left(\gamma_{m} W^{12}\right)_{\alpha}+k_{m}^{12} A_{\alpha}^{12}+\left(k^{1} \cdot k^{2}\right)\left(A_{\alpha}^{1} A_{m}^{2}-A_{\alpha}^{2} A_{m}^{1}\right) \\
D_{\alpha} W_{12}^{\beta} & =\frac{1}{4}\left(\gamma^{m n}\right)_{\alpha}{ }^{\beta} F_{m n}^{12}+\left(k^{1} \cdot k^{2}\right)\left(A_{\alpha}^{1} W_{2}^{\beta}-A_{\alpha}^{2} W_{1}^{\beta}\right)
\end{aligned}
$$

\footnotetext{
${ }^{9}$ In string calculations this cancellation involves a subtle interplay of BRST-exact terms and total derivatives on the worldsheet, see [45] and [57] for five- and six-point examples at tree level. One manifestation is the agreement of the superfields along with $\partial_{1}, \partial_{2}$ in (3.4) with the BRST-exact admixtures in $V_{1}\left(z_{1}\right) U_{2}\left(z_{2}\right) \rightarrow z_{12}^{-k^{1} \cdot k^{2}-1}\left(V_{12}+Q\left[\left(A_{1} W_{2}\right)-\frac{1}{2}\left(A_{1} \cdot A_{2}\right)\right]\right)$.
} 


$$
\begin{aligned}
D_{\alpha} F_{m n}^{12}= & k_{m}^{12}\left(\gamma_{n} W^{12}\right)_{\alpha}-k_{n}^{12}\left(\gamma_{m} W^{12}\right)_{\alpha}+\left(k^{1} \cdot k^{2}\right)\left(A_{\alpha}^{1} F_{m n}^{2}-A_{\alpha}^{2} F_{m n}^{1}\right) \\
& +\left(k^{1} \cdot k^{2}\right)\left(A_{n}^{1}\left(\gamma_{m} W^{2}\right)_{\alpha}-A_{n}^{2}\left(\gamma_{m} W^{1}\right)_{\alpha}-A_{m}^{1}\left(\gamma_{n} W^{2}\right)_{\alpha}+A_{m}^{2}\left(\gamma_{n} W^{1}\right)_{\alpha}\right),
\end{aligned}
$$

which is a clear generalization of the standard equations of motion (2.1) with corrections proportional to the conformal weight $\sim \frac{1}{2}\left(k^{1}+k^{2}\right)^{2}=\left(k^{1} \cdot k^{2}\right)$ of the superfields. Furthermore, the single-particle relations $k^{m} A_{m}^{i}=0$ and $k_{m}\left(\gamma^{m} W_{i}\right)_{\alpha}=0$ imply that,

$$
\begin{aligned}
k_{12}^{m} A_{m}^{12} & =0 \\
k_{m}^{12}\left(\gamma^{m} W^{12}\right)_{\alpha} & =\left(k^{1} \cdot k^{2}\right)\left[A_{m}^{1}\left(\gamma^{m} W^{2}\right)_{\alpha}-(1 \leftrightarrow 2)\right] \\
k_{12}^{m} F_{m n}^{12} & =\left(k^{1} \cdot k^{2}\right)\left[A_{n}^{12}+A_{n}^{1}\left(k^{1} \cdot A^{2}\right)-(1 \leftrightarrow 2)\right] .
\end{aligned}
$$

In other words, the (supersymmetrized) Dirac and YM equations $k_{m}^{i}\left(\gamma^{m} W^{i}\right)_{\alpha}=0$ and $k_{i}^{m} F_{m n}^{i}=0$ for single-particle superfields are modified by the same kind of contact term $\sim\left(k^{1} \cdot k^{2}\right)$ as the field strength relation in (3.5) and the equations of motion (3.7) to (3.10).

Defining the rank-two unintegrated vertex operator as

$$
V^{12}=\lambda^{\alpha} A_{\alpha}^{12}
$$

analogously to $V^{i}=\lambda^{\alpha} A_{\alpha}^{i}$, one can show that

$$
\begin{aligned}
& Q V^{12}=\left(k^{1} \cdot k^{2}\right) V_{1} V_{2} \\
& Q U^{12}=\partial V^{12}+\left(k^{1} \cdot k^{2}\right)\left(V^{1} U^{2}-V^{2} U^{1}\right),
\end{aligned}
$$

which generalizes (2.4) by contact terms and reproduces the BRST variation of the building block $T_{12}$ of [12]. It is interesting to note that (3.16) is compatible with the standard prescription relating integrated and unintegrated vertices, $U^{12}=b_{-1} V^{12}[25]$.

Note that all rank-two BRST blocks are antisymmetric and therefore $U^{12}=-U^{21}$.

\subsection{Rank three}

Since the rank-two BRST blocks obey generalized SYM equations of motion one is tempted to define the rank-three BRST blocks following a similar approach. We know from (2.1) that the standard superfields $A_{m}, W^{\alpha}$ and $F^{m n}$ can be obtained from the spinor superpotential $A_{\alpha}$ by recursively computing covariant derivatives. We will show that the a similar approach can be used to obtain their multiparticle generalizations starting from the following ansatz for the superpotential,

$$
\hat{A}_{\alpha}^{123}=-\frac{1}{2}\left[A_{\alpha}^{12}\left(k^{12} \cdot A^{3}\right)+A_{m}^{12}\left(\gamma^{m} W^{3}\right)_{\alpha}-(12 \leftrightarrow 3)\right] .
$$

This is a direct generalization of the expression for $A_{\alpha}^{12}$ in (3.5) as obtained from the OPE of $U^{1}\left(z_{1}\right) U^{2}\left(z_{2}\right)$. We have now inserted two-particle data represented by $A_{\alpha}^{12}, k_{m}^{12}, A_{m}^{12}$ and $W_{12}^{\alpha}$ into the OPE-inspired recursion. Once the BRST-trivial symmetry components are subtracted from $\hat{A}_{\alpha}^{123}$ (see section 3.2.1), the definition (3.17) can be interpreted in terms of a "grafting" procedure defined for example in [4]. As illustrated in figure 3, (3.17) amounts 

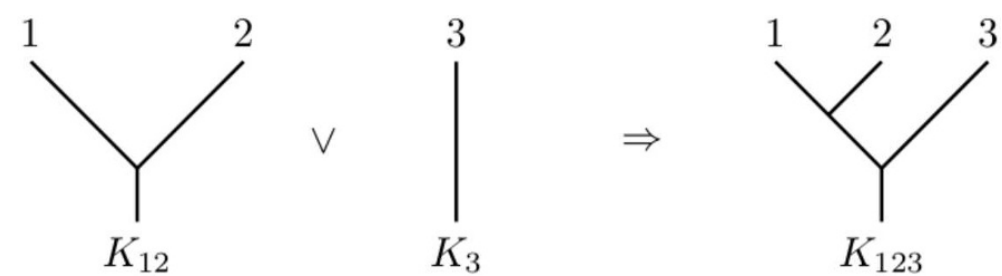

Figure 3. The essentials of the first rank three BRST block $K_{123} \in\left\{A_{\alpha}^{123}, \ldots\right\}$ are captured by combining $K_{12} \in\left\{A_{\alpha}^{12}, A_{12}^{m}, W_{12}^{\alpha}\right\}$ and $K_{3} \in\left\{A_{\alpha}^{3}, A_{3}^{m}, W_{3}^{\alpha}\right\}$. At the level of diagrams, this can be interpreted as grafting the trees associated with $K_{12}$ and $K_{3}$.

to adjoining a further leg to the cubic graph associated with the BRST blocks $K_{12}$ at rank two, see appendix A for more details.

A short computation shows that the action of the covariant derivative can be written in a form similar to (3.8) and therefore can be used to define $\hat{A}_{123}^{m}$,

$$
\begin{aligned}
D_{\alpha} \hat{A}_{\beta}^{123}+D_{\beta} \hat{A}_{\alpha}^{123}= & \gamma_{\alpha \beta}^{m} \hat{A}_{m}^{123} \\
& +\left(k^{1} \cdot k^{2}\right)\left[A_{\alpha}^{1} A_{\beta}^{23}+A_{\alpha}^{13} A_{\beta}^{2}-(1 \leftrightarrow 2)\right] \\
& +\left(k^{12} \cdot k^{3}\right)\left[A_{\alpha}^{12} A_{\beta}^{3}-(12 \leftrightarrow 3)\right]
\end{aligned}
$$

where

$$
\hat{A}_{m}^{123}=\frac{1}{2}\left[A_{12}^{p} F_{p m}^{3}-A_{m}^{12}\left(k^{12} \cdot A^{3}\right)+\left(W^{12} \gamma_{m} W^{3}\right)-(12 \leftrightarrow 3)\right] .
$$

In turn, computing the covariant derivative of (3.19) and rewriting the result in a form analogous to the standard equation of motion for $A^{m}$ leads to the definition of $W_{123}^{\alpha}$,

$$
\begin{aligned}
D_{\alpha} \hat{A}_{m}^{123}= & \left(\gamma_{m} W^{123}\right)_{\alpha}+k_{m}^{123} \hat{A}_{\alpha}^{123} \\
& +\left(k^{1} \cdot k^{2}\right)\left[A_{\alpha}^{1} A_{m}^{23}+A_{\alpha}^{13} A_{m}^{2}-A_{\alpha}^{23} A_{m}^{1}-A_{\alpha}^{2} A_{m}^{13}\right] \\
& +\left(k^{12} \cdot k^{3}\right)\left(A_{\alpha}^{12} A_{m}^{3}-A_{\alpha}^{3} A_{m}^{12}\right)
\end{aligned}
$$

where

$$
\begin{aligned}
W_{123}^{\alpha}= & -\left(k^{12} \cdot A^{3}\right) W_{12}^{\alpha}+\frac{1}{4}\left(\gamma^{r s} W^{3}\right)^{\alpha} F_{r s}^{12}-(12 \leftrightarrow 3) \\
& +\frac{1}{2}\left(k^{1} \cdot k^{2}\right)\left[W_{2}^{\alpha}\left(A^{1} \cdot A^{3}\right)-(1 \leftrightarrow 2)\right] .
\end{aligned}
$$

Computing the covariant derivative of (3.21) leads to the definition of $F_{m n}^{123}$,

$$
\begin{aligned}
D_{\alpha} W_{123}^{\beta}= & \frac{1}{4}\left(\gamma^{m n}\right)_{\alpha}{ }^{\beta} F_{m n}^{123} \\
& +\left(k^{1} \cdot k^{2}\right)\left[A_{\alpha}^{1} W_{23}^{\beta}+A_{\alpha}^{13} W_{2}^{\beta}-(1 \leftrightarrow 2)\right] \\
& +\left(k^{12} \cdot k^{3}\right)\left[A_{\alpha}^{12} W_{3}^{\beta}-(12 \leftrightarrow 3)\right],
\end{aligned}
$$

where (3.12) has been used to arrive at,

$$
\begin{aligned}
F_{m n}^{123}= & \left(k^{3} \cdot A^{12}\right) F_{m n}^{3}+F_{a[m}^{12} F_{n] a}^{3}+2 k_{[m}^{12}\left(W^{3} \gamma_{n]} W^{12}\right)-(12 \leftrightarrow 3) \\
& +\left(k^{1} \cdot k^{2}\right)\left[\frac{1}{2} F_{m n}^{2}\left(A^{1} \cdot A^{3}\right)+2 A_{[m}^{1}\left(W^{3} \gamma_{n]} W^{2}\right)-(1 \leftrightarrow 2)\right] .
\end{aligned}
$$


And finally,

$$
\begin{aligned}
D_{\alpha} F_{m n}^{123}= & 2 k_{[m}^{123}\left(\gamma_{n]} W^{123}\right)_{\alpha} \\
& +\left(k^{1} \cdot k^{2}\right)\left[A_{\alpha}^{1} F_{m n}^{23}+A_{\alpha}^{13} F_{m n}^{2}-(1 \leftrightarrow 2)\right] \\
& +\left(k^{12} \cdot k^{3}\right)\left[A_{\alpha}^{12} F_{m n}^{3}-(12 \leftrightarrow 3)\right] \\
& +\left(k^{1} \cdot k^{2}\right)\left[2 A_{[n}^{1}\left(\gamma_{m]} W^{23}\right)_{\alpha}+2 A_{[n}^{13}\left(\gamma_{m]} W^{2}\right)_{\alpha}-(1 \leftrightarrow 2)\right] \\
& +\left(k^{12} \cdot k^{3}\right)\left[2 A_{[n}^{12}\left(\gamma_{m]} W^{3}\right)_{\alpha}-(12 \leftrightarrow 3)\right] .
\end{aligned}
$$

The above equations give rise to a natural rank-three definition of multiparticle SYM equations of motion: the non-contact terms in (3.18), (3.20), (3.22) and (3.24) perfectly tie in with those in the two-particle equations of motion (3.7) to (3.10). Note that the contact terms in $D_{\alpha} A_{123}^{m}$ and $D_{\alpha} W_{123}^{\beta}$ are related via $A_{C}^{m} \leftrightarrow W_{C}^{\alpha}$ where $C$ denotes a multiparticle label, see (3.20) and (3.22). The additional contact terms of the form $A_{[n}^{B}\left(\gamma_{m]} W^{C}\right)$ in $D_{\alpha} F_{m n}^{123}$ have their two-particle analogues in the second line of (3.10).

\subsubsection{Symmetry properties at rank three}

The rank-three superfields defined above are manifestly antisymmetric in the first two labels, so they satisfy $£_{2}$ from (3.1). However, one can show using the explicit expressions above that only a subset of the rank-three superfields satisfies $£_{3}$,

$$
£_{3} \circ \hat{A}_{\alpha}^{123} \neq 0, \quad £_{3} \circ \hat{A}_{m}^{123} \neq 0, \quad £_{3} \circ W_{123}^{\alpha}=£_{3} \circ F_{m n}^{123}=0 .
$$

This explains the non-hatted notation for $W_{123}^{\alpha}$ and $F_{m n}^{123}$; they are BRST blocks already. To obtain BRST blocks for the other superfields they need to be redefined in order to satisfy the symmetry $£_{3}$. Fortunately, the underlying system of equations of motion greatly simplifies this task.

To see this, note that since $£_{3} \circ W_{123}^{\alpha}=0$ equation (3.20) implies that,

$$
D_{\alpha}\left(£_{3} \circ \hat{A}_{m}^{123}\right)=k_{m}^{123}\left(£_{3} \circ \hat{A}_{\alpha}^{123}\right) .
$$

And it turns out that $k_{m}^{123}$ can be factored out in the cyclic sum of $\hat{A}_{m}^{123}$,

$$
£_{3} \circ \hat{A}_{123}^{m}=3 k_{123}^{m} H_{123},
$$

where

$$
H^{123}=\frac{1}{6}\left[\left(A^{1} \cdot A^{23}\right)-\left(k_{p}^{2}-k_{p}^{3}\right) A_{1}^{p}\left(A^{2} \cdot A^{3}\right)+\operatorname{cyclic}(123)\right] .
$$

Therefore the redefinitions

$$
\begin{aligned}
& A_{m}^{123}=\hat{A}_{m}^{123}-k_{m}^{123} H^{123}, \\
& A_{\alpha}^{123}=\hat{A}_{\alpha}^{123}-D_{\alpha} H^{123},
\end{aligned}
$$

imply that $A_{\alpha}^{123}$ and $A_{m}^{123}$ are BRST blocks since,

$$
£_{2} \circ A_{\alpha}^{123}=£_{2} \circ A_{m}^{123}=£_{3} \circ A_{\alpha}^{123}=£_{3} \circ A_{m}^{123}=0 .
$$


This is a significant simplification compared to the redefinition (2.8). The latter required an "inversion" of the BRST charge on $£_{3} \circ\left(L_{2131}+\ldots\right)$ whereas $(3.27)$ extracts the rankthree redefinition $H_{123}$ from a straightforward $£_{3}$ operation on the known expression (3.19) for $\hat{A}_{123}^{m}$.

It is easy to show that $F_{m n}^{123}$ from (3.23) can now be rewritten as a field-strength using the BRST block $A_{m}^{123}$,

$$
\begin{aligned}
F_{m n}^{123}= & k_{m}^{123} A_{n}^{123}-k_{n}^{123} A_{m}^{123} \\
& -\left(k^{1} \cdot k^{2}\right)\left[A_{m}^{1} A_{n}^{23}-A_{n}^{1} A_{m}^{23}-(1 \leftrightarrow 2)\right] \\
& -\left(k^{12} \cdot k^{3}\right)\left[A_{m}^{12} A_{n}^{3}-(12 \leftrightarrow 3)\right] .
\end{aligned}
$$

Thus (3.23) satisfying the symmetry $£_{3} \circ F^{123}=0$ can be understood as a property inherited from $A_{123}^{m}$ since the contact term structure of (3.30) is the same as in the equation of motion $D_{\alpha} A_{m}^{123}$ from which the BRST symmetry was derived in the first place.

Defining rank-three vertex operators

$$
V_{123}=\lambda^{\alpha} A_{\alpha}^{123}, \quad U^{123}=\partial \theta^{\alpha} A_{\alpha}^{123}+\Pi^{m} A_{m}^{123}+d_{\alpha} W_{123}^{\alpha}+\frac{1}{2} N^{m n} F_{m n}^{123},
$$

it follows that (2.4) as well as (3.15) and (3.16) have a rank-three counterpart,

$$
\begin{aligned}
Q V_{123}= & \left(k^{1} \cdot k^{2}\right)\left(V_{1} V_{23}+V_{13} V_{2}\right)+\left(k^{12} \cdot k^{3}\right) V_{12} V_{3}, \\
Q U_{123}= & \partial V_{123}+\left(k^{1} \cdot k^{2}\right)\left[V_{1} U_{23}+V_{13} U_{2}-(1 \leftrightarrow 2)\right] \\
& +\left(k^{12} \cdot k^{3}\right)\left[V_{12} U_{3}-(12 \leftrightarrow 3)\right] .
\end{aligned}
$$

It is interesting to observe that $£_{3}$ action translates to a total derivative

$$
\hat{U}^{123}+\hat{U}^{231}+\hat{U}^{312}=\left(\partial \theta^{\alpha} D_{\alpha}+\Pi^{m} k_{m}^{123}\right) H^{123}=\partial H^{123}
$$

where $\hat{U}^{123}$ is related to $U^{123}$ in the obvious way $A_{\alpha}^{123} \leftrightarrow \hat{A}_{\alpha}^{123}$ and $A_{m}^{123} \leftrightarrow \hat{A}_{m}^{123}$. The total worldsheet derivative suggests that the failure of the $£_{3}$ symmetries in (3.34) decouples from string amplitudes and their SYM limit. In view of the diagrammatic interpretation of $K_{123}$ shown in figure 3 , the vanishing of $U^{123}+U^{231}+U^{312}$ can be viewed as the kinematic dual of the Jacobi identity $f^{12 a} f^{a 3 b}+f^{23 a} f^{a 1 b}+f^{31 a} f^{a 2 b}=0$ among color factors. This indicates that the rank three superfields $K_{123}$ of SYM carry the fingerprints of the BCJ duality between color and kinematics [26].

\subsection{Rank four}

The patterns from the discussions above suggest how to proceed. The following superfields

$$
\begin{aligned}
\hat{A}_{\alpha}^{1234} & =-\frac{1}{2}\left[A_{\alpha}^{123}\left(k^{123} \cdot A^{4}\right)+A_{m}^{123}\left(\gamma^{m} W^{4}\right)_{\alpha}-(123 \leftrightarrow 4)\right], \\
\hat{A}_{m}^{1234} & =\frac{1}{2}\left[A_{p}^{123} F_{p m}^{4}-A_{m}^{123}\left(k^{123} \cdot A^{4}\right)+\left(W^{123} \gamma_{m} W^{4}\right)-(123 \leftrightarrow 4)\right] \\
\hat{W}_{1234}^{\alpha} & =\frac{1}{4}\left(\gamma^{r s} W^{4}\right)^{\alpha} F_{r s}^{123}-\left(k^{123} \cdot A^{4}\right) W_{123}^{\alpha}-(123 \leftrightarrow 4)
\end{aligned}
$$



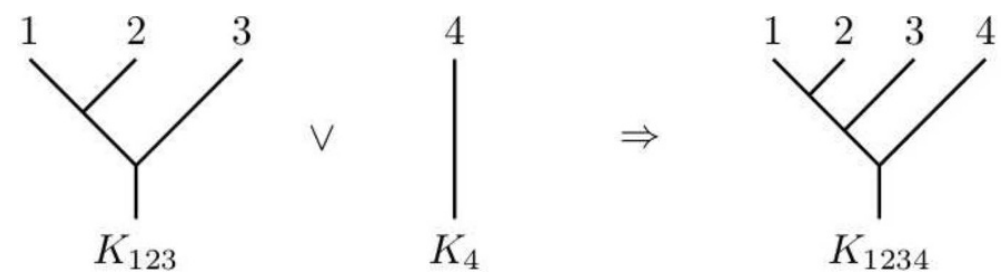

Figure 4. Up to $£_{4}$ symmetry redefinitions, the recursions (3.35) to (3.37) yield rank-four BRST blocks $K_{1234}$ by combining $K_{123}$ with $K_{4}$. At the level of diagrams, this can be interpreted as grafting the associated trees.

$$
\begin{aligned}
& +\frac{1}{2}\left(k^{1} \cdot k^{2}\right)\left[W_{23}^{\alpha}\left(A^{1} \cdot A^{4}\right)+W_{2}^{\alpha}\left(A^{13} \cdot A^{4}\right)-(1 \leftrightarrow 2)\right] \\
& +\frac{1}{2}\left(k^{12} \cdot k^{3}\right)\left[W_{3}^{\alpha}\left(A^{12} \cdot A^{4}\right)-(12 \leftrightarrow 3)\right]
\end{aligned}
$$

manifestly satisfy the $£_{2}$ and $£_{3}$ symmetries of (3.1). In general, by using the fully redefined BRST-blocks $A_{\alpha}^{12 \ldots p-1}, A_{12 \ldots p-1}^{m}$ and $W_{12 \ldots p-1}^{\alpha}$ in the recursive definition of $A_{\alpha}^{12 \ldots p}$, there is only one novel Lie symmetry to impose at each rank. This is much more economic compared to the $p-1$ redefinitions to arrive at $T_{12 \ldots p}$ in $[13,14]$ (which additionally required "inverting" the BRST charge and were much more laborious). Once the last Lie symmetry $£_{4}$ is enforced in section 3.3.1, the recursions (3.35) to (3.37) for $K_{1234}$ can be given a grafting interpretation similar to rank three, see figure 4 and appendix $\mathrm{A}$.

The rank-four definitions (3.35) to (3.37) are guided by the same key principles applied at rank three: repetition of the recursive pattern (3.17), (3.19) and (3.21) as well as multiparticle equations of motion as in (3.18), (3.20) and (3.22). Straightforward but tedious calculations show that

$$
\begin{aligned}
& D_{\alpha} \hat{A}_{\beta}^{1234}+D_{\beta} \hat{A}_{\alpha}^{1234}=\gamma_{\alpha \beta}^{m} \hat{A}_{m}^{1234} \\
& +\left(k^{1} \cdot k^{2}\right)\left[A_{\alpha}^{1} \hat{A}_{\beta}^{234}+\hat{A}_{\alpha}^{134} A_{\beta}^{2}+A_{\alpha}^{13} A_{\beta}^{24}+A_{\alpha}^{14} A_{\beta}^{23}-(1 \leftrightarrow 2)\right] \\
& +\left(k^{12} \cdot k^{3}\right)\left[A_{\alpha}^{12} A_{\beta}^{34}+\hat{A}_{\alpha}^{124} A_{\beta}^{3}-(12 \leftrightarrow 3)\right] \\
& +\left(k^{123} \cdot k^{4}\right)\left[A_{\alpha}^{123} A_{\beta}^{4}-(123 \leftrightarrow 4)\right] \\
& D_{\alpha} \hat{A}_{m}^{1234}=\left(\gamma_{m} \hat{W}^{1234}\right)_{\alpha}+k_{m}^{1234} \hat{A}_{\alpha}^{1234} \\
& +\left(k^{1} \cdot k^{2}\right)\left[A_{\alpha}^{1} \hat{A}_{m}^{234}+\hat{A}_{\alpha}^{134} A_{m}^{2}+A_{\alpha}^{13} A_{m}^{24}+A_{\alpha}^{14} A_{m}^{23}-(1 \leftrightarrow 2)\right] \\
& +\left(k^{12} \cdot k^{3}\right)\left[A_{\alpha}^{12} A_{m}^{34}+\hat{A}_{\alpha}^{124} A_{m}^{3}-(12 \leftrightarrow 3)\right] \\
& +\left(k^{123} \cdot k^{4}\right)\left[A_{\alpha}^{123} A_{m}^{4}-(123 \leftrightarrow 4)\right] \\
& D_{\alpha} \hat{W}_{1234}^{\beta}=\frac{1}{4}\left(\gamma^{m n}\right)_{\alpha}{ }^{\beta} \hat{F}_{m n}^{1234} \\
& +\left(k^{1} \cdot k^{2}\right)\left[A_{\alpha}^{1} W_{234}^{\beta}+\hat{A}_{\alpha}^{134} W_{2}^{\beta}+A_{\alpha}^{13} W_{24}^{\beta}+A_{\alpha}^{14} W_{23}^{\beta}-(1 \leftrightarrow 2)\right] \\
& +\left(k^{12} \cdot k^{3}\right)\left[A_{\alpha}^{12} W_{34}^{\beta}+\hat{A}_{\alpha}^{124} W_{3}^{\beta}-(12 \leftrightarrow 3)\right] \\
& +\left(k^{123} \cdot k^{4}\right)\left[A_{\alpha}^{123} W_{4}^{\beta}-(123 \leftrightarrow 4)\right]
\end{aligned}
$$


for some $\hat{F}_{m n}^{1234}$ whose form is not important at this point. Note that the rank-three superfields in the terms proportional to $\left(k^{123} \cdot k^{4}\right)$ are the true BRST blocks and not their hatted versions.

\subsubsection{Symmetry properties at rank four}

The hatted superfields appearing in the right-hand side of (3.38) to (3.40) can be rewritten in terms of BRST blocks by using the rank three redefinitions $\hat{A}_{\alpha}^{123}=A_{\alpha}^{123}+D_{\alpha} H_{123}$ and $\hat{A}_{m}^{123}=A_{m}^{123}+k_{m}^{123} H_{123}$. The terms containing $H_{i j k}$ can be manipulated to the left-hand side in order to redefine the rank-four superfields. The outcome is,

$$
K_{1234}^{\prime}=\hat{K}_{1234}-\left(k^{1} \cdot k^{2}\right)\left(K_{2} H_{134}-K_{1} H_{234}\right)-\left(k^{12} \cdot k^{3}\right) H_{124} K_{3}
$$

where $K^{B}$ denotes any of the BRST blocks, $\left[A_{\alpha}^{B}, A_{m}^{B}, W_{B}^{\alpha}\right]$. For example,

$$
A_{\alpha}^{\prime 1234}=\hat{A}_{\alpha}^{1234}-\left(k^{1} \cdot k^{2}\right)\left(A_{\alpha}^{2} H_{134}-A_{\alpha}^{1} H_{234}\right)-\left(k^{12} \cdot k^{3}\right) H_{124} A_{\alpha}^{3} .
$$

After the redefinitions of (3.41) it turns out that the superfield $W_{1234}^{\prime \alpha}$ satisfies all the Lie symmetries (3.1) up to rank four,

$$
£_{2} \circ W_{1234}^{\prime \alpha}=£_{3} \circ W_{1234}^{\prime \alpha}=£_{4} \circ W_{1234}^{\prime \alpha}=0,
$$

and therefore $W_{1234}^{\alpha} \equiv W_{1234}^{\prime \alpha}$ is a BRST block.

Since $W_{1234}^{\alpha}$ satisfies (3.43), it immediately follows from the contact term structure of (3.39) that (3.26) has the following rank-four analogue

$$
D_{\alpha}\left(£_{4} \circ A_{m}^{\prime 1234}\right)=k_{m}^{1234}\left(£_{4} \circ A_{\alpha}^{\prime 1234}\right) .
$$

Furthermore, a straightforward calculation shows that $k_{m}^{1234}$ factorizes in $£_{4} \circ A_{m}^{\prime 1234}$,

$$
£_{4} \circ A_{m}^{\prime 1234}=4 k_{m}^{1234} H^{1234},
$$

and the explicit expression for $H_{1234}$ is displayed in appendix $\mathrm{C}$.

Hence, the redefined superfields

$$
\begin{aligned}
& A_{m}^{1234}=A_{m}^{\prime 1234}-k_{m}^{1234} H^{1234} \\
& A_{\alpha}^{1234}=A_{\alpha}^{\prime 1234}-D_{\alpha} H^{1234}
\end{aligned}
$$

obey the required BRST symmetries:

$$
\begin{aligned}
& £_{2} \circ A_{\alpha}^{1234}=£_{3} \circ A_{\alpha}^{1234}=£_{4} \circ A_{\alpha}^{1234}=0, \\
& £_{2} \circ A_{m}^{1234}=£_{3} \circ A_{m}^{1234}=£_{4} \circ A_{m}^{1234}=0,
\end{aligned}
$$

and therefore define rank-four BRST blocks.

Once the expression for $A_{1234}^{m}$ is known the superfield $F_{m n}^{1234}$ can be written down immediately in field-strength form,

$$
\begin{aligned}
F_{m n}^{1234}= & k_{m}^{1234} A_{n}^{1234}-k_{n}^{1234} A_{m}^{1234} \\
& +\left(k^{1} \cdot k^{2}\right)\left[A_{n}^{1} A_{m}^{234}+A_{n}^{134} A_{m}^{2}+A_{n}^{13} A_{m}^{24}+A_{n}^{14} A_{m}^{23}-(m \leftrightarrow n)\right] \\
& +\left(k^{12} \cdot k^{3}\right)\left[A_{n}^{12} A_{m}^{34}+A_{n}^{124} A_{m}^{3}-(m \leftrightarrow n)\right] \\
& +\left(k^{123} \cdot k^{4}\right)\left[A_{n}^{123} A_{m}^{4}-A_{m}^{123} A_{n}^{4}\right] .
\end{aligned}
$$


A straightforward but tedious calculation then shows that its expected equation of motion indeed holds,

$$
\begin{aligned}
D_{\alpha} F_{m n}^{1234}= & k_{m}^{1234}\left(\gamma_{n} W^{1234}\right)_{\alpha}-k_{n}^{1234}\left(\gamma_{m} W^{1234}\right)_{\alpha} \\
& +\left(k^{1} \cdot k^{2}\right)\left[A_{\alpha}^{1} F_{m n}^{234}+A_{\alpha}^{134} F_{m n}^{2}+A_{\alpha}^{13} F_{m n}^{24}+A_{\alpha}^{14} F_{m n}^{23}-(1 \leftrightarrow 2)\right] \\
& +\left(k^{12} \cdot k^{3}\right)\left[A_{\alpha}^{12} F_{m n}^{34}+A_{\alpha}^{124} F_{m n}^{3}-(12 \leftrightarrow 3)\right] \\
& +\left(k^{123} \cdot k^{4}\right)\left[A_{\alpha}^{123} F_{m n}^{4}-A_{\alpha}^{4} F_{m n}^{123}\right] \\
& +\left(k^{1} \cdot k^{2}\right)\left[2 A_{[n}^{1}\left(\gamma_{m]} W^{234}\right)_{\alpha}+2 A_{[n}^{134}\left(\gamma_{m]} W^{2}\right)_{\alpha}\right. \\
& \left.\quad+2 A_{[n}^{14}\left(\gamma_{m]} W^{23}\right)_{\alpha}+2 A_{[n}^{13}\left(\gamma_{m]} W^{24}\right)_{\alpha}-(1 \leftrightarrow 2)\right] \\
& +\left(k^{12} \cdot k^{3}\right)\left[2 A_{[n}^{12}\left(\gamma_{m]} W^{34}\right)_{\alpha}+2 A_{[n}^{124}\left(\gamma_{m]} W^{3}\right)_{\alpha}-(12 \leftrightarrow 3)\right] \\
& +\left(k^{123} \cdot k^{4}\right)\left[2 A_{[n}^{123}\left(\gamma_{m]} W^{4}\right)_{\alpha}-(123 \leftrightarrow 4)\right] .
\end{aligned}
$$

That is why the explicit form of $\hat{F}_{1234}^{m n}$ was not strictly needed, one can directly write its BRST-block expression at the end of the redefinition procedure.

Defining rank-four vertex operators

$$
V^{1234}=\lambda^{\alpha} A_{\alpha}^{1234}, \quad U^{1234}=\partial \theta^{\alpha} A_{\alpha}^{1234}+\Pi^{m} A_{m}^{1234}+d_{\alpha} W_{1234}^{\alpha}+\frac{1}{2} N^{m n} F_{m n}^{1234},
$$

it follows that

$$
\begin{aligned}
Q V_{1234}= & \left(k^{1} \cdot k^{2}\right)\left[V_{1} V_{234}+V_{134} V_{2}+V_{13} V_{24}+V_{14} V_{23}\right] \\
& +\left(k^{12} \cdot k^{3}\right)\left[V_{12} V_{34}+V_{124} V_{3}\right] \\
& +\left(k^{123} \cdot k^{4}\right) V_{123} V_{4} \\
Q U_{1234}= & \partial V_{1234}+\left(k^{1} \cdot k^{2}\right)\left[V_{1} U_{234}+V_{13} U_{24}+V_{14} U_{23}+V_{134} U_{2}-(1 \leftrightarrow 2)\right] \\
& +\left(k^{12} \cdot k^{3}\right)\left[V_{12} U_{34}+V_{124} U_{3}-(12 \leftrightarrow 3)\right] \\
& +\left(k^{123} \cdot k^{4}\right)\left[V_{123} U_{4}-(123 \leftrightarrow 4)\right] .
\end{aligned}
$$

And similarly as at rank three, it is interesting that the failure of the $£_{4}$ symmetry to hold for the primed superfields is equivalent to a total derivative in the integrated vertex $U^{\prime 1234}$ (i.e. $U^{1234}$ with $A_{\alpha}^{1234} \rightarrow A_{\alpha}^{\prime 1234}$ and $A_{m}^{1234} \rightarrow A_{m}^{\prime 1234}$ ). Due to the general expectation for worldsheet derivatives to decouple from string amplitudes, this is another example for the fundamental role played by Lie symmetries. More specifically, $£_{4}$ compatibility of $U^{1234}$ is a kinematic equivalent of Jacobi identities among permutations of $f^{12 a} f^{a 3 b} f^{b 4 c}$. Hence, also the rank four BRST blocks satisfying $£_{4} \circ K_{1234}=0$ point towards the BCJ-duality [26].

\subsection{Recursive construction at general rank}

Suppose that all the BRST blocks up to rank $p-1$ are known

$$
\left\{A_{\alpha}^{12 \ldots k}, A_{m}^{12 \ldots k}, W_{12 \ldots k}^{\alpha}, F_{m n}^{12 \ldots k}\right\}, \quad 1 \leq k \leq p-1
$$

together with the superfields $H_{12 \ldots k}$ for $3 \leq k \leq p-1$ used in their construction. The following steps can be used to obtain the explicit expressions for the rank- $p$ BRST blocks: 
1. Define a set of rank-p superfields $\hat{K}_{12 \ldots p}=\left\{\hat{A}_{\alpha}^{12 \ldots p-1}, \hat{A}_{m}^{12 \ldots p-1}, \hat{W}_{12 \ldots p-1}^{\alpha}\right\}$ as follows,

$$
\begin{aligned}
\hat{A}_{\alpha}^{12 \ldots p}= & -\frac{1}{2}\left[A_{\alpha}^{12 \ldots p-1}\left(k^{12 \ldots p-1} \cdot A^{p}\right)+A_{m}^{12 \ldots p-1}\left(\gamma^{m} W^{p}\right)_{\alpha}-(12 \ldots p-1 \leftrightarrow p)\right] \\
\hat{A}_{m}^{12 \ldots p}= & \frac{1}{2}\left[A_{n}^{12 \ldots p-1} F_{n m}^{p}+A_{m}^{p}\left(k^{p} \cdot A^{12 \ldots p-1}\right)+\left(W^{12 \ldots p-1} \gamma_{m} W^{p}\right)-(12 \ldots p-1 \leftrightarrow p)\right] \\
\hat{W}_{12 \ldots p}^{\alpha}= & \frac{1}{4}\left(\gamma^{r s} W^{p}\right)^{\alpha} F_{r s}^{12 \ldots p-1}-\left(k^{12 \ldots p-1} \cdot A^{p}\right) W_{12 \ldots p-1}^{\alpha}-(12 \ldots p-1 \leftrightarrow p) \\
& -\sum_{j=2}^{p-1} \sum_{\delta \in P\left(\gamma_{j}\right)}\left(k^{1 \ldots j-1} \cdot k^{j}\right)\left[W_{1 \ldots j-1,\{\delta\}}^{\alpha}\left(A^{j,\left\{\gamma_{j} \backslash \delta\right\}} \cdot A^{p}\right)-(12 \ldots j-1 \leftrightarrow j)\right],
\end{aligned}
$$

where the set $\gamma_{j}=\{j+1, \ldots, p-1\}$ contains the $p-j-1$ labels between $j$ and $p$ and $P\left(\gamma_{j}\right)$ is its power set. Note that they manifestly obey all the $£_{k}$ symmetries up to rank $k=p-1$, but not (yet) $£_{p}$.

One can check that the superfields $\hat{K}_{12 \ldots p}$ satisfy equations of motion of the form (3.61) whose right-hand side contains not only lower-rank BRST blocks but also their hatted versions, for example,

$$
\begin{aligned}
2 D_{(\alpha} \hat{A}_{\beta)}^{12345}= & \gamma_{\alpha \beta}^{m} \hat{A}_{m}^{12345} \\
& +\left(k^{1} \cdot k^{2}\right)\left[A_{\alpha}^{1} \hat{A}_{\beta}^{2345}+A_{\alpha}^{13} \hat{A}_{\beta}^{245}+A_{\alpha}^{14} \hat{A}_{\beta}^{235}+A_{\alpha}^{15} A_{\beta}^{234}\right. \\
& \left.\quad+A_{\alpha}^{134} A_{\beta}^{25}+\hat{A}_{\alpha}^{135} A_{\beta}^{24}+\hat{A}_{\alpha}^{145} A_{\beta}^{23}+\hat{A}_{\alpha}^{1345} A_{\beta}^{2}-(1 \leftrightarrow 2)\right] \\
& +\left(k^{12} \cdot k^{3}\right)\left[A_{\alpha}^{12} \hat{A}_{\beta}^{345}+A_{\alpha}^{124} A_{\beta}^{35}+\hat{A}_{\alpha}^{125} A_{\beta}^{34}+\hat{A}_{\alpha}^{1245} A_{\beta}^{3}-(12 \leftrightarrow 3)\right] \\
& +\left(k^{123} \cdot k^{4}\right)\left[A_{\alpha}^{123} A_{\beta}^{45}+\hat{A}_{\alpha}^{1235} A_{\beta}^{4}-(123 \leftrightarrow 4)\right] \\
& +\left(k^{1234} \cdot k^{5}\right)\left[A_{\alpha}^{1234} A_{\beta}^{5}-(1234 \leftrightarrow 5)\right] .
\end{aligned}
$$

However, they can be redefined $\hat{K}_{12 \ldots p} \rightarrow K_{12 \ldots p}^{\prime}$ such that equations of motion for $K_{12 \ldots p}^{\prime}$ are written entirely in terms of BRST blocks with rank less than $p$. This leads to the second step:

2. Redefine the superfields according to

$$
K_{12 \ldots p}^{\prime}=\hat{K}_{12 \ldots p}-\sum_{j=2}^{p-1} \sum_{\delta \in P\left(\gamma_{j}\right)}\left(k^{1 \ldots j-1} \cdot k^{j}\right)\left[H_{1 \ldots j-1,\{\delta\}, p} K_{j,\left\{\gamma_{j} \backslash \delta\right\}}-(12 \ldots j-1 \leftrightarrow j)\right]
$$

with the constraints $H_{i}=H_{i j}=0$. For example,

$$
\begin{aligned}
K_{12345}^{\prime}= & \hat{K}_{12345} \\
& -\left(k^{1} \cdot k^{2}\right)\left[H_{1345} K_{2}+H_{145} K_{23}+H_{135} K_{24}-(1 \leftrightarrow 2)\right] \\
& -\left(k^{12} \cdot k^{3}\right)\left[H_{1245} K_{3}+H_{125} K_{34}-H_{345} K_{12}\right] \\
& -\left(k^{123} \cdot k^{4}\right)\left[H_{1235} K_{4}\right] .
\end{aligned}
$$

At this point it turns out that $W_{12 \ldots p}^{\prime \alpha}$ satisfies all the rank- $p$ Lie symmetries, i.e.

$$
£_{k} \circ W_{12 \ldots p}^{\prime \alpha}=0, \quad 2 \leq k \leq p .
$$


Therefore $W_{12 \ldots p}^{\prime \alpha} \equiv W_{12 \ldots p}^{\alpha}$ will be the definition of the spinor field-strength BRST block.

As a consequence of (3.58), the following equations will hold,

$$
\begin{aligned}
D_{\alpha}\left(£_{p} \circ A_{m}^{\prime 12 \ldots p}\right) & =k_{m}^{12 \ldots p} £_{p} \circ A_{\alpha}^{\prime 12 \ldots p}, \\
£_{p} \circ A_{12 \ldots p}^{\prime m} & =p k_{12 \ldots p}^{m} H_{12 \ldots p}
\end{aligned}
$$

where the second equation can be regarded as the definition of $H_{12 \ldots p}$.

3. The rank- $p$ BRST blocks are defined as,

$$
\begin{aligned}
A_{\alpha}^{12 \ldots p} & =A_{\alpha}^{12 \ldots p}-D_{\alpha} H^{12 \ldots p} \\
A_{m}^{12 \ldots p} & =A_{m}^{12 \ldots p}-k_{m}^{12 \ldots p} H^{12 \ldots p} \\
W_{12 \ldots p}^{\alpha} & =W_{12 \ldots p}^{\prime \alpha} \\
F_{m n}^{12 \ldots p} & =k_{m}^{12 \ldots p} A_{n}^{12 \ldots p}-k_{n}^{12 \ldots p} A_{m}^{12 \ldots p} \\
& \quad+\sum_{j=2}^{p} \sum_{\delta \in P\left(\beta_{j}\right)}\left(k_{12 \ldots j-1} \cdot k_{j}\right) 2 A_{[n}^{12 \ldots j-1,\{\delta\}} A_{m]}^{j,\left\{\beta_{j} \backslash \delta\right\}},
\end{aligned}
$$

where the set $\beta_{j}=\{j+1, j+2, \ldots, p\}$ contains the $p-j$ labels to the right of $j$ and $P\left(\beta_{j}\right)$ denotes its power set. Note that they satisfy all the Lie symmetries up to rank $p$.

It is conjectured that the BRST blocks defined in the three-step procedure above will satisfy the multiparticle equations of motion,

$$
\begin{aligned}
2 D_{(\alpha} A_{\beta)}^{12 \ldots p}= & \gamma_{\alpha \beta}^{m} A_{m}^{12 \ldots p} \\
& +\sum_{j=2}^{p} \sum_{\delta \in P\left(\beta_{j}\right)}\left(k_{12 \ldots j-1} \cdot k_{j}\right)\left[A_{\alpha}^{12 \ldots j-1,\{\delta\}} A_{\beta}^{j,\left\{\beta_{j} \backslash \delta\right\}}-(12 \ldots j-1 \leftrightarrow j)\right] \\
D_{\alpha} A_{12 \ldots p}^{m}= & \left(\gamma^{m} W_{12 \ldots p}\right)_{\alpha}+k_{12 \ldots p}^{m} A_{\alpha}^{12 \ldots p} \\
& +\sum_{j=2}^{p} \sum_{\delta \in P\left(\beta_{j}\right)}\left(k_{12 \ldots j-1} \cdot k_{j}\right)\left[A_{\alpha}^{12 \ldots j-1,\{\delta\}} A_{j,\left\{\beta_{j} \backslash \delta\right\}}^{m}-(12 \ldots j-1 \leftrightarrow j)\right] \\
D_{\alpha} W_{12 \ldots p}^{\beta}= & \frac{1}{4}\left(\gamma^{m n}\right)_{\alpha}^{\beta} F_{m n}^{12 \ldots p} \\
& +\sum_{j=2}^{p} \sum_{\delta \in P\left(\beta_{j}\right)}\left(k_{12 \ldots j-1} \cdot k_{j}\right)\left[A_{\alpha}^{12 \ldots j-1,\{\delta\}} W_{j,\left\{\beta_{j} \backslash \delta\right\}}^{\beta}-(12 \ldots j-1 \leftrightarrow j)\right] \\
D_{\alpha} F_{12 \ldots p}^{m n}=2 & k_{12 \ldots p}^{[m}\left(\gamma^{n]} W_{12 \ldots p}\right)_{\alpha} \\
& +\sum_{j=2}^{p} \sum_{\delta \in P\left(\beta_{j}\right)}\left(k_{12 \ldots j-1} \cdot k_{j}\right)\left[A_{\alpha}^{12 \ldots j-1,\{\delta\}} F_{j,\left\{\beta_{j} \backslash \delta\right\}}^{m n}\right. \\
& \left.+2 A_{12 \ldots j-1,\{\delta\}}^{[n}\left(\gamma^{m]} W_{j,\left\{\beta_{j} \backslash \delta\right\}}\right)_{\alpha}-(12 \ldots j-1 \leftrightarrow j)\right] .
\end{aligned}
$$


Furthermore, defining the multiparticle vertex operators as

$$
V^{B}=\lambda^{\alpha} A_{\alpha}^{B}, \quad U^{B}=\partial \theta^{\alpha} A_{\alpha}^{B}+\Pi^{m} A_{m}^{B}+d_{\alpha} W_{B}^{\alpha}+\frac{1}{2} N^{m n} F_{m n}^{B},
$$

one can show using the equations of motion (3.61) that they satisfy

$$
\begin{aligned}
& Q V_{12 \ldots p}= \sum_{j=2}^{p} \sum_{\alpha \in P\left(\beta_{j}\right)}\left(k^{12 \ldots j-1} \cdot k^{j}\right) V_{12 \ldots j-1,\{\alpha\}} V_{j,\left\{\beta_{j} \backslash \alpha\right\}}, \\
& Q U_{12 \ldots p}=\partial V_{12 \ldots p}+\sum_{j=2}^{p} \sum_{\alpha \in P\left(\beta_{j}\right)}\left(k^{12 \ldots j-1} \cdot k^{j}\right) \\
& \quad\left[V_{12 \ldots j-1,\{\alpha\}} U_{j,\left\{\beta_{j} \backslash \alpha\right\}}-(12 \ldots j-1 \leftrightarrow j)\right] .
\end{aligned}
$$

It is interesting to note that there is an alternative definition ${ }^{10}$ of the rank- $p$ BRST blocks $A_{\alpha}^{12 \ldots p}$ and $A_{m}^{12 \ldots p}$ in (3.60) which does not require the explicit knowledge of the rank- $p H_{12 \ldots p}$ (assuming it exists). One can simply project $A_{\alpha}^{\prime 12 \ldots p}$ and $A_{m}^{\prime 12 \ldots p}$ into the kernel of $£_{p^{\circ}}$, for example, use $\frac{3}{4} A_{m}^{\prime 1234}+\frac{1}{4}\left(A_{m}^{\prime 1243}-A_{m}^{\prime 3412}+A_{m}^{\prime 3421}\right)$ rather than (3.46) as a definition for $A_{m}^{1234}$ and similarly for $A_{\alpha}^{1234}$. This is convenient since it allows to get the complete set of rank $p$ BRST blocks using $H_{12 \ldots k}$ with $k \leq p-1$.

We have explicitly constructed BRST blocks up to rank four using the steps above. Furthermore, preliminary checks also indicate that this construction works for rank five.

\section{Berends-Giele currents}

In the 1980's, Berends and Giele introduced the concept of gluonic tree amplitudes with one off-shell leg and found a recursive construction for these so-called "currents" [9]. Physical amplitudes are easily recovered by removing the off-shell propagator (as represented by the dots in figure 5) from the current. In the following, we construct ten-dimensional superspace representations of Berends-Giele currents from multiparticle SYM superfields. The particular combinations of rank- $p$ superfields is firstly guided by the cubic diagrams of a $p+1$ tree amplitude. Secondly, it turns out that the contact terms of their multiparticle equations of motion (3.61) simplify when following the diagrammatic intuition.

This construction has been partially realized in [12] for the superpotential $A_{\alpha}^{12 \ldots p}$ which suffices to determine the SYM tree amplitude from a supersymmetric Berends-Giele recursion. In the superspace setup, the divergent off-shell propagator is cancelled by the BRST charge, see section 5.1. At one-loop level [10], Berends-Giele currents from the field strengths $W_{12 \ldots p}^{\alpha}, F_{12 \ldots p}^{m n}$ were assembled to BRST-invariant kinematic factors. We shall now provide a unified discussion of all the Berends-Giele currents associated with the multiparticle superfields of the previous section.

For each multiparticle superfield $K_{B} \in\left\{A_{\alpha}^{B}, A_{B}^{m}, W_{B}^{\alpha}, F_{B}^{m n}\right\}$ with $B=12 \ldots p$ we define a ghost-number zero Berends-Giele current $\mathcal{K}_{B} \in\left\{\mathcal{A}_{\alpha}^{B}, \mathcal{A}_{B}^{m}, \mathcal{W}_{B}^{\alpha}, \mathcal{F}_{B}^{m n}\right\}$ as follows:

\footnotetext{
${ }^{10}$ In fact, this is the representation chosen in all the checks performed with a computer.
} 

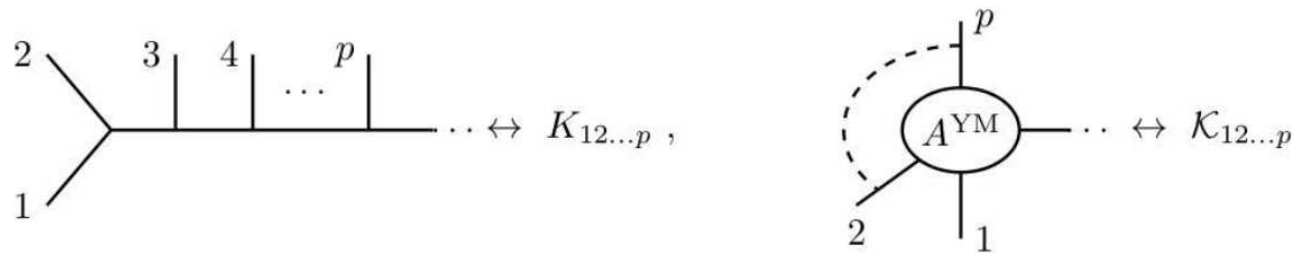

Figure 5. From cubic diagrams $K_{A}$ to Berends-Giele currents $\mathcal{K}_{A}$.

firstly decorate the cubic diagrams represented by $K$ with their propagators and secondly combine the propagator-dressed diagrams such that they resemble a color-ordered YangMills tree amplitude with an off-shell leg [9], see figure $5 .{ }^{11}$ As pointed out in [29], this is implemented through the inverse momentum kernel $[27,28]^{12}$

$$
\mathcal{K}_{1 \sigma(23 \ldots p)} \equiv \sum_{\rho \in S_{p-1}} S^{-1}[\sigma \mid \rho]_{1} K_{1 \rho(23 \ldots p)}
$$

where $\sigma \in S_{p-1}$, and the momentum kernel $S[\cdot \mid \cdot]_{1}$ is defined as

$$
S\left[2_{\rho}, \ldots, p_{\rho} \mid 2_{\sigma}, \ldots, p_{\sigma}\right]_{1} \equiv \prod_{j=2}^{p}\left(s_{1, j_{\rho}}+\sum_{k=2}^{j-1} \theta\left(j_{\rho}, k_{\rho}\right) s_{j_{\rho}, k_{\rho}}\right) .
$$

We use the shorthands $s_{i j}=k^{i} \cdot k^{j}$ and $i_{\rho} \equiv \rho(i)$, and the object $\theta\left(j_{\rho}, k_{\rho}\right)$ equals 1 (zero) if the ordering of the legs $j_{\rho}, k_{\rho}$ is the same (opposite) in the ordered sets $\rho(2, \ldots, p)$ and $\sigma(2, \ldots, p)$. In other words, it keeps track of labels which swap their relative positions in the two permutations $\rho$ and $\sigma$. At rank $r \leq 4$, for example,

$$
\begin{aligned}
\mathcal{K}_{12} & =\frac{K_{12}}{s_{12}}, \quad \mathcal{K}_{123}=\frac{K_{123}}{s_{12} s_{123}}+\frac{K_{321}}{s_{23} s_{123}}, \\
\mathcal{K}_{1234} & =\frac{1}{s_{1234}}\left(\frac{K_{1234}}{s_{12} s_{123}}+\frac{K_{3214}}{s_{23} s_{123}}+\frac{K_{12[34]}}{s_{12} s_{34}}+\frac{K_{3421}}{s_{34} s_{234}}+\frac{K_{3241}}{s_{23} s_{234}}\right),
\end{aligned}
$$

and figure 6 illustrates that the given expression for $\mathcal{K}_{1234}$ reproduces the five cubic diagrams in a color-ordered SYM five-point amplitude with an off-shell leg.

The ghost-number zero Berends-Giele currents $\mathcal{K} \in\left\{\mathcal{A}_{\alpha}^{12 \ldots p}, \mathcal{A}_{12 \ldots p}^{m}, \mathcal{W}_{12 \ldots p}^{\alpha}, \mathcal{F}_{12 \ldots p}^{m n}\right\}$ generalize the ghost-number one analogues $M_{12 \ldots p}$ studied in [12-14] which correspond to the unintegrated multiparticle vertex as

$$
\mathcal{V}_{A} \equiv \lambda^{\alpha} \mathcal{A}_{\alpha}^{A} \equiv M_{A} .
$$

One can show using the equations of motion (3.61) that the BRST charge acts on BerendsGiele currents of any ghost number by simple deconcatenation of labels

$$
Q M_{12 \ldots p}=\sum_{j=1}^{p-1} M_{12 \ldots j} M_{j+1 \ldots p}
$$

\footnotetext{
${ }^{11}$ See appendix A.3 for a more mathematical approach to this diagrammatic construction.

${ }^{12}$ In the conventions of $[29], S[\sigma \mid \rho]_{1}$ is symmetric under exchange of $\sigma$ and $\rho$. For example, the rank two and three versions of its inverse are given by

$$
S^{-1}[2 \mid 2]_{1}=\frac{1}{s_{12}}, \quad S^{-1}[23 \mid 23]_{1}=\frac{1}{s_{12} s_{123}}+\frac{1}{s_{123} s_{23}}, \quad S^{-1}[23 \mid 32]_{1}=-\frac{1}{s_{123} s_{23}} .
$$
}




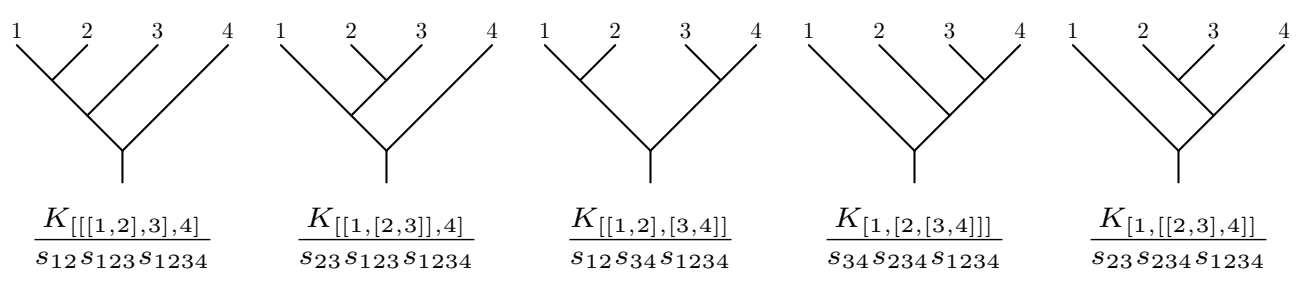

Figure 6. The Berends-Giele current $\mathcal{K}_{1234}$ of (4.2) is given by the sum of the superspace expressions associated with the above five cubic graphs with one leg off-shell. The mapping between the cubic graphs and BRST blocks is introduced in section 3, figure 2 and explained in more detail in appendix A.

as well as

$$
\begin{gathered}
Q \mathcal{A}_{12 \ldots p}^{m}=\left(\lambda \gamma^{m} \mathcal{W}_{12 \ldots p}\right)+k_{12 \ldots p}^{m} \mathcal{V}_{12 \ldots p}+\sum_{j=1}^{p-1}\left(\mathcal{V}_{12 \ldots j} \mathcal{A}_{j+1 \ldots p}^{m}-\mathcal{V}_{j+1 \ldots p} \mathcal{A}_{12 \ldots j}^{m}\right) \\
Q \mathcal{W}_{12 \ldots p}^{\alpha}=\frac{1}{4}\left(\lambda \gamma_{m n}\right)^{\alpha} \mathcal{F}_{12 \ldots p}^{m n}+\sum_{j=1}^{p-1}\left(\mathcal{V}_{12 \ldots j} \mathcal{W}_{j+1 \ldots p}^{\alpha}-\mathcal{V}_{j+1 \ldots p} \mathcal{W}_{12 \ldots j}^{\alpha}\right) \\
Q \mathcal{F}_{12 \ldots p}^{m n}=2 k_{12 \ldots p}^{[m}\left(\lambda \gamma^{n]} \mathcal{W}_{12 \ldots p}\right)+\sum_{j=1}^{p-1}\left(\mathcal{V}_{12 \ldots j} \mathcal{F}_{j+1 \ldots p}^{m n}-\mathcal{V}_{j+1 \ldots p} \mathcal{F}_{12 \ldots j}^{m n}\right) \\
+\sum_{j=1}^{p-1} 2\left[\mathcal{A}_{12 \ldots j}^{[n}\left(\lambda \gamma^{m]} \mathcal{W}_{j+1 \ldots p}\right)-\mathcal{A}_{j+1 \ldots p}^{[n}\left(\lambda \gamma^{m]} \mathcal{W}_{12 \ldots j}\right)\right] .
\end{gathered}
$$

By comparing the above equations with (3.61) one sees that the kinematic poles in the definition of the Berends-Giele currents absorb all the explicit kinematic invariants $\left(k^{12 \ldots j-1} \cdot k^{j}\right)$ from the right-hand side of the BRST variations. The extra simplicity of (4.5) and (4.6) compared to (3.61) suggests that the Berends-Giele basis of tree subdiagrams is particularly suitable for a systematic construction of BRST-invariants, see section 5.

\subsection{Symmetries of Berends-Giele currents}

Under the momentum kernel multiplication (4.1), the Lie-symmetries of the multiparticle superfields $K_{12 \ldots p}$ are mapped to a different set of Berends-Giele symmetries of $\mathcal{K}_{12 \ldots p}$,

$$
\mathcal{K}_{12}+\mathcal{K}_{21}=0, \quad \mathcal{K}_{123}-\mathcal{K}_{321}=\mathcal{K}_{123}+\mathcal{K}_{231}+\mathcal{K}_{312}=0, \quad \ldots
$$

which leave the same number $(p-1)$ ! of independent components at rank $p$. Universality of the momentum kernel implies that any of the $\mathcal{K}_{12 \ldots p}$ shares the same symmetry properties as $M_{12 \ldots p}$ discussed in [12-14], namely ${ }^{13}$

$$
\mathcal{K}_{\{\beta\}, 1,\{\alpha\}}=(-1)^{n_{\beta}} \mathcal{K}_{1, \alpha \uplus \beta^{T}}
$$

The notation $\left\{\beta^{T}\right\}$ represents the set with the reversed ordering of its $n_{\beta}$ elements and $ш$ denotes the shuffle product. Furthermore, the convention $\mathcal{K}_{\ldots \alpha \omega \beta \ldots} \equiv \sum_{\sigma \in \alpha \omega \beta} \mathcal{K}_{\ldots\{\sigma\} \ldots}$ has

\footnotetext{
${ }^{13}$ As a consequence, we have $\mathcal{K}_{\alpha \amalg \beta}=0, \forall \alpha, \beta$.
} 
been used. The multiparticle label $B$ in $\mathcal{K}_{B}$ now carries Berends-Giele symmetries (4.7) rather than the Lie symmetries (3.1) of the associated $K_{B}$.

The symmetry properties (4.7) of rank- $p$ currents can be viewed as rank- $(p+1)$ KleissKuijf relation [30,31] obeyed by Yang-Mills tree amplitudes where the last leg $p+1$ is off-shell and not displayed, leaving $(p-1)$ ! independent components. Note, however, that the off-shell-ness of one leg in the diagrammatic interpretation of Berends-Giele currents obstructs an analogue of the BCJ relations [26] among Yang-Mills tree amplitudes.

On the other hand, an interesting perspective on BCJ relations is opened up when the recursions (3.54) for BRST blocks are rewritten in terms of Berends-Giele currents. This observation is presented in appendix B, which leads to a simplified rewriting of one-loop kinematics in terms of SYM amplitudes as compared to [10].

\section{Application to the one loop cohomology}

In this section, we explore examples at one-loop how the universal multiparticle equations of motions (3.61) and the simplified contact terms in the Berends-Giele picture (4.5) and (4.6) facilitate the construction of BRST invariants. The scalar BRST cohomology at one-loop has been investigated in [10] and identified in the non-anomalous part of open string amplitudes. The trial-and-error construction of the invariants' expansion in terms of Berends-Giele currents is now replaced by a clean recursion. The same mechanisms are applied to novel vectorial invariants which play a key role in closed string amplitudes at one loop, e.g. for S-duality [20] and for loop momentum dependence in the numerators of the field theory limit [21].

\subsection{Tree level SYM amplitudes}

As shown in [12], tree amplitudes $A^{\mathrm{YM}}$ of ten-dimensional SYM theory take an elegant form in pure spinor superspace,

$$
A^{\mathrm{YM}}(1,2, \ldots, n)=\left\langle E_{12 \ldots n-1} V_{n}\right\rangle .
$$

The central object $E_{12 \ldots n-1}$ belongs to the BRST cohomology in the momentum phase space of $n$ massless particles. ${ }^{14}$ Its explicit form can be written in terms of the BerendsGiele currents associated with the (generalized) unintegrated vertex $V_{A}$ as follows,

$$
E_{12 \ldots p}=\sum_{j=1}^{p-1} M_{12 \ldots j} M_{j+1 \ldots p} .
$$

The pure spinor bracket $\langle\ldots\rangle$ in (5.1) denotes a zero-mode integration prescription of schematic form $\left\langle\lambda^{3} \theta^{5}\right\rangle=1$. It extracts the gluon and gluino components of the enclosed superfields [17] as has been automated in [32]. The explicit form of the SYM amplitudes in terms of polarization vectors and gaugino wavefunctions up to multiplicity eight can be downloaded from [46].

\footnotetext{
${ }^{14}$ The restriction on the momentum phase space follows from the fact that the solution $M_{12 \ldots n-1}$ in $E_{12 \ldots n-1}=Q M_{12 \ldots n-1}$ is proportional to a divergent propagator $s_{12 \ldots n-1}^{-1}$.
} 
The BRST cohomology techniques that were used in [12] to cast the SYM scattering amplitudes into the form (5.1) also played a crucial role in obtaining the general solution of the $n$-point tree-level amplitude of massless open superstrings [13, 14].

\subsection{Scalar BRST blocks at one-loop}

In [10] the pure spinor zero-mode saturation rules in one-loop amplitudes of the open superstring were used to obtain an effective prescription to identify contributing pure spinor superspace expressions: the zero modes of $d_{\alpha} d_{\beta} N^{m n}$ extracted from the external vertices are replaced by $\left(\lambda \gamma^{[m}\right)_{\alpha}\left(\lambda \gamma^{n]}\right)_{\beta}$. This prescription leads to the BRST-closed expression $\left(\lambda \gamma^{m} W^{i}\right)\left(\lambda \gamma^{n} W^{j}\right) F_{m n}^{k}$ in the four-point amplitude [19] and motivates the following higherpoint definitions, ${ }^{15}$

$$
\begin{aligned}
T_{A, B, C} & \equiv \frac{1}{3}\left(\lambda \gamma_{m} W_{A}\right)\left(\lambda \gamma_{n} W_{B}\right) F_{C}^{m n}+(C \leftrightarrow A, B), \\
M_{A, B, C} & \equiv \frac{1}{3}\left(\lambda \gamma_{m} \mathcal{W}_{A}\right)\left(\lambda \gamma_{n} \mathcal{W}_{B}\right) \mathcal{F}_{C}^{m n}+(C \leftrightarrow A, B) .
\end{aligned}
$$

Using the universal form of $Q \mathcal{W}_{B}^{\alpha}$ and $Q \mathcal{F}_{B}^{m n}$, one sees that the BRST variation of (5.4) is given by deconcatenation of the multiparticle indices. Regardless of the ranks $|A|,|B|$ and $|C|$, the pure spinor constraint projects out all terms in (4.6) with an explicit appearance of $\lambda^{\alpha}$, and we are left with the BRST-covariant expression

$$
Q M_{A, B, C}=\sum_{\ell=1}^{|A|-1}\left(M_{a_{1} \ldots a_{\ell}} M_{a_{\ell+1} \ldots a_{|A|}, B, C}-M_{a_{\ell+1} \ldots a_{|A|}} M_{a_{1} \ldots a_{\ell}, B, C}\right)+(A \leftrightarrow B, C) .
$$

Note that $Q T_{1,2,3}=Q M_{1,2,3}=0$ and that $T_{A, B, C}$ and $M_{A, B, C}$ are totally symmetric in $A$, $B$ and $C$.

\subsection{Scalar BRST cohomology at one-loop}

The definition (5.4) of building blocks $M_{A, B, C}$ was used in [10] to construct BRST invariants $C_{1 \mid A, B, C}$ with up to eight particles by trial and error. We will now present a recursive method to generate them for arbitrary ranks.

The results of [10] suggest that each term of the form $M_{i} M_{A, B, C}$, with $i$ a single-particle label, can be completed to a BRST-closed expression of the schematic form

$$
C_{i \mid A, B, C} \equiv M_{i} M_{A, B, C}+\sum_{\{\delta\} \neq \emptyset} M_{i\{\delta\}} f_{\{\delta\}}\left(M_{\cdot,,, .}\right) .
$$

As a defining property of the BRST completion for $M_{i} M_{A, B, C}$, particle $i$ always enters in a multiparticle Berends-Giele current $M_{D}$. This is formally represented by a sum over (non-empty) ordered subsets $\{\delta\}$ of the labels $\left\{a_{i}\right\},\left\{b_{i}\right\},\left\{c_{i}\right\}$ in $A, B, C$ which join particle $i$ in $M_{i\{\delta\}}$. The functions $f_{\{\delta\}}$ represent the accompanying linear combinations of building blocks $M_{A, B, C}$.

\footnotetext{
${ }^{15} T_{A, B, C}$ and $M_{A, B, C}$ were denoted by $T_{A}^{i} T_{B}^{j} T_{C}^{k}$ and $M_{A}^{i} M_{B}^{j} M_{C}^{k}$ in [10], and the representation of $W_{A}$ and $F_{B}$ given in the reference is different from the current setup.
} 
Nilpotency $Q^{2}=0$ implies that $Q M_{A, B, C}$ is also BRST closed, and the form of (5.5) suggests that it can be expanded as

$$
Q M_{A, B, C}=C_{a_{1} \mid a_{2} \ldots a_{|A|}, B, C}-C_{a_{|A|} \mid a_{1} \ldots a_{|A|-1}, B, C}+(A \leftrightarrow B, C) .
$$

We have picked up all the terms $M_{i} M_{D, E, F}$ in (5.5) with single-particle label $i$ and promoted them to BRST completions $C_{i \mid D, E, F}$. Examples of (5.7) can indeed be checked to hold once the explicit expressions for $C_{i \mid D, E, F}$ are generated. At five points for instance, $C_{1 \mid 23,4,5}=$ $M_{1} M_{23,4,5}+M_{12} M_{3,4,5}-M_{13} M_{2,4,5}$ (to be derived shortly) allows to verify

$$
\begin{aligned}
Q M_{123,4,5} & =M_{1} M_{23,4,5}+M_{12} M_{3,4,5}-M_{23} M_{1,4,5}-M_{3} M_{12,4,5} \\
& =C_{1 \mid 23,4,5}-C_{3 \mid 12,4,5} .
\end{aligned}
$$

Now we turn towards the explicit construction of the BRST completion $f_{\{\delta\}}\left(M_{,,,},\right)$in (5.6). The task is to cancel terms like $M_{i}\left(C_{a_{|A|} \mid a_{1} \ldots a_{|A|-1}, B, C}-C_{a_{1} \mid a_{2} \ldots a_{|A|}, B, C}\right)$ as they appear in $Q\left(M_{i} M_{A, B, C}\right)$ by (5.7). In order to determine $f_{\{\delta\}}\left(M_{,,, .}\right)$with this property, we define a linear concatenation operation $\otimes_{j}$ acting on the multiparticle labels of Berends-Giele currents $M_{A}$ as follows,

$$
M_{i} \otimes_{a_{1}} M_{a_{1} a_{2} \ldots a_{|A|}} \equiv M_{i a_{1} a_{2} \ldots a_{|A|}} .
$$

In order to ensure that the concatenation $\otimes_{a_{1}}$ preserves the KK symmetries $M_{a_{1} a_{2} \ldots a_{|A|}}$ of the Berends-Giele currents, we have to specify the leg $a_{1}$ appearing next to the concatenating label $i$ on the right hand side: for example, $M_{132} \neq-M_{123}$ implies that $M_{1} \otimes_{3} M_{32} \neq-M_{1} \otimes_{2} M_{23}$ even though $M_{32}=-M_{23}$. The definition (5.9) would be inconsistent with linearity of $\otimes_{j}$ if the subscript $j$ is unspecified. The $\otimes_{j}$ action on additional $M_{B, C, D}$ building blocks is defined to be trivial,

$$
M_{i} \otimes_{a_{1}}\left(M_{a_{1} a_{2} \ldots a_{|A|}} M_{B, C, D}\right) \equiv\left(M_{i} \otimes_{a_{1}} M_{a_{1} a_{2} \ldots a_{|A|}}\right) M_{B, C, D}
$$

As we will see in the following Lemma, there is a neat interplay between action of the BRST charge and the $\otimes_{j}$ operation defined in (5.9).

Lemma 1. If $C_{j \mid A, B, C}$ as defined by (5.6) is BRST closed, then its concatenation satisfies

$$
Q\left(M_{i} \otimes_{j} C_{j \mid A, B, C}\right)=M_{i} C_{j \mid A, B, C} .
$$

For example, $C_{2 \mid 3,4,5}=M_{2} M_{3,4,5}$ is BRST closed and $M_{1} \otimes_{2} C_{2 \mid 3,4,5}=M_{12} M_{3,4,5}$ satisfies $Q\left(M_{1} \otimes_{2} C_{2 \mid 3,4,5}\right)=M_{1} M_{2} M_{3,4,5}=M_{1} C_{2 \mid 3,4,5}$.

Proof. BRST closure of $C_{j \mid A, B, C}$ amounts to the following ghost number four statement

$$
Q\left(C_{j \mid A, B, C}\right)=\sum_{\{\sigma\}} M_{j\{\sigma\}} F_{\{\sigma\}}\left(M . M_{,,, .}\right)=0
$$

with linear combinations $F_{\{\sigma\}}$ of ghost number three objects $M_{.} M_{,,,}$, .. Since $M_{j\{\sigma\}}$ are independent for different sets $\{\sigma\}$, the $F_{\{\sigma\}}$ must vanish individually. Using the deconcate- 
nation formula (4.5), one can rewrite the left hand side of (5.10) as follows:

$$
\begin{aligned}
Q\left(M_{i} \otimes_{j} C_{j \mid A, B, C}\right) & =Q\left(M_{i j} M_{A, B, C}+\sum_{\{\delta\} \neq \emptyset} M_{i j\{\delta\}} f_{\{\delta\}}\left(M_{.,, .}\right)\right) \\
& =M_{i} M_{j} M_{A, B, C}+\sum_{\{\delta\} \neq \emptyset} M_{i} M_{j\{\delta\}} f_{\{\delta\}}\left(M_{., .,}\right)+\sum_{\{\sigma\}} M_{i j\{\sigma\}} F_{\{\sigma\}}\left(M_{.} M_{., .,}\right) \\
& =M_{i}\left\{M_{j} M_{A, B, C}+\sum_{\{\delta\} \neq \emptyset} M_{j\{\delta\}} f_{\{\delta\}}\left(M_{\cdot, \cdot, \cdot)}\right\}\right. \\
& =M_{i} C_{j \mid A, B, C} .
\end{aligned}
$$

In the first step, we have isolated the first term of $Q M_{i j\{\delta\}}=M_{i} M_{j\{\delta\}}+\ldots$ and the second step made use of $F_{\{\sigma\}}=0 \forall\{\sigma\}$ as argued above.

The following recursive definition can be checked to generate BRST closed expressions for arbitrary ranks

$C_{i \mid A, B, C}=M_{i} M_{A, B, C}+\left[M_{i} \otimes_{a_{1}} C_{a_{1} \mid a_{2} \ldots a_{|A|}, B, C}-M_{i} \otimes_{a_{|A|}} C_{a_{|A|} \mid a_{1} \ldots a_{|A|-1}, B, C}+(A \leftrightarrow B, C)\right]$.

$Q$-invariance follows from (5.7) and Lemma 1 (using the definition $C_{1 \mid \emptyset, A, B}=0$ for singleparticle slots). The are $7-2 k$ terms in (5.12) where $k$ is the number of single-particle slots among $A, B, C$. Since $M_{i} \otimes_{j}$ increases the multiplicity of $C_{j \mid D, E, F}$ on the right hand side by one, we can regard (5.12) as a recursion in $|A|+|B|+|C|$. Its first applications up to multiplicity $1+|A|+|B|+|C|=6$ are listed below

$$
\begin{aligned}
& C_{1 \mid 2,3,4} \equiv M_{1} M_{2,3,4} \\
& C_{1 \mid 23,4,5} \equiv M_{1} M_{23,4,5}+M_{1} \otimes_{2} C_{2 \mid 3,4,5}-M_{1} \otimes_{3} C_{3 \mid 2,4,5} \\
&= M_{1} M_{23,4,5}+M_{12} M_{3,4,5}-M_{13} M_{2,4,5} \\
& C_{1 \mid 234,5,6} \equiv M_{1} M_{234,5,6}+M_{1} \otimes_{2} C_{2 \mid 34,5,6}-M_{1} \otimes_{4} C_{4 \mid 23,5,6} \\
&=M_{1} M_{234,5,6}+M_{12} M_{34,5,6}+M_{123} M_{4,5,6}-M_{124} M_{3,5,6} \\
& \quad \quad-M_{14} M_{23,5,6}-M_{142} M_{3,5,6}+M_{143} M_{2,5,6} \\
& C_{1 \mid 23,45,6} \equiv M_{1} M_{23,45,6}+M_{1} \otimes_{2} C_{2 \mid 45,3,6}-M_{1} \otimes_{3} C_{3 \mid 45,2,6}+M_{1} \otimes_{4} C_{4 \mid 23,5,6}-M_{1} \otimes_{5} C_{5 \mid 23,4,6} \\
&=M_{1} M_{23,45,6}+M_{12} M_{45,3,6}-M_{13} M_{45,2,6}+M_{14} M_{23,5,6}-M_{15} M_{23,4,6} \\
& \quad \quad \quad \quad M_{124} M_{3,5,6}-M_{134} M_{2,5,6}+M_{142} M_{3,5,6}-M_{152} M_{3,4,6} \\
& \quad-M_{125} M_{3,4,6}+M_{135} M_{2,4,6}-M_{143} M_{2,5,6}+M_{153} M_{2,4,6}
\end{aligned}
$$

and higher-rank expressions are easily obtained as well. Even though the number of terms in $C_{1 \mid 234,5,6}$ and $C_{1 \mid 23,45,6}$ can be reduced by virtue of the Berends-Giele symmetry $M_{124}+$ $M_{142}=-M_{214}$, we keep the expression in the form $M_{1 \ldots}$ compatible with further recursion steps (5.12).

As detailed in appendix $\mathrm{B}$, the $C_{1 \mid A, B, C}$ boil down to linear combinations of SYM tree amplitudes [10]. Nevertheless, their component expansion up to multiplicity seven can be downloaded from [46]. 


\subsection{Vector BRST blocks at one-loop}

In the five-point closed string computation of [20] the zero mode saturation in the left/rightmixing sector where the b-ghost contributes $\Pi^{m} d_{\alpha} d_{\beta}$ led to the definition

$$
W_{2,3,4,5}^{m} \equiv \frac{1}{12}\left(\lambda \gamma^{n} W^{2}\right)\left(\lambda \gamma^{p} W^{3}\right)\left(W^{4} \gamma^{m n p} W^{5}\right)+(2,3 \mid 2,3,4,5),
$$

which satisfies

$$
Q W_{2,3,4,5}^{m}=-\left(\lambda \gamma^{m} W_{2}\right) T_{3,4,5}-(2 \leftrightarrow 3,4,5) .
$$

The notation $\left(i_{1}, i_{2} \mid i_{1}, \ldots, i_{n}\right)$ means a sum over all possible ways of choosing two indices $i_{1}$ and $i_{2}$ out of $i_{1}, \ldots, i_{n}$, for a total of $\left(\begin{array}{l}n \\ 2\end{array}\right)$ terms. Furthermore, another type of left/rightmixing zero-mode saturation was possible which required taking $\Pi^{m} d_{\alpha} d_{\beta} N_{n p}$ from the integrated vertex operators, leading to terms of the form $A_{2}^{m} T_{3,4,5}$. The key observation in [20] was that the vectorial superfield

$$
T_{2,3,4,5}^{m} \equiv A_{2}^{m} T_{3,4,5}+(2 \leftrightarrow 3,4,5)+W_{2,3,4,5}^{m}
$$

has a BRST variation in which the vector index is carried only by momenta

$$
Q T_{2,3,4,5}^{m}=k_{2}^{m} V_{2} T_{3,4,5}+(2 \leftrightarrow 3,4,5) .
$$

This fact played a crucial role in demonstrating BRST invariance of the closed-string fivepoint amplitude [20] because it allows the BRST variation of the terms contracting leftand right-movers to factorize and cancel the variation of the holomorphic squared terms.

To generalize this construction to higher multiplicity one defines

$$
\begin{aligned}
W_{A, B, C, D}^{m} & \equiv \frac{1}{12}\left(\lambda \gamma^{n} W_{A}\right)\left(\lambda \gamma^{p} W_{B}\right)\left(W_{C} \gamma^{m n p} W_{D}\right)+(A, B \mid A, B, C, D) \\
T_{A, B, C, D}^{m} & \equiv A_{A}^{m} T_{B, C, D}+(A \leftrightarrow B, C, D)+W_{A, B, C, D}^{m}
\end{aligned}
$$

with multiparticle labels $A, B, C, D$ as well as their Berends-Giele counterparts,

$$
\begin{aligned}
\mathcal{W}_{A, B, C, D}^{m} & \equiv \frac{1}{12}\left(\lambda \gamma^{n} \mathcal{W}_{A}\right)\left(\lambda \gamma^{p} \mathcal{W}_{B}\right)\left(\mathcal{W}_{C} \gamma^{m n p} \mathcal{W}_{D}\right)+(A, B \mid A, B, C, D) \\
M_{A, B, C, D}^{m} & \equiv \mathcal{A}_{A}^{m} M_{B, C, D}+(A \leftrightarrow B, C, D)+\mathcal{W}_{A, B, C, D}^{m},
\end{aligned}
$$

which are totally symmetric in $A, B, C, D$. The BRST variations (4.6) - in particular the universality of the non-contact terms to arbitrary $A, B, C$ and $D$ - imply that

$$
\begin{aligned}
Q \mathcal{W}_{A, B, C, D}^{m}= & -\left(\lambda \gamma^{m} \mathcal{W}_{A}\right) M_{B, C, D} \\
& +\sum_{j=1}^{|A|-1}\left(M_{a_{1} \ldots a_{j}} \mathcal{W}_{a_{j+1} \ldots a_{|A|}, B, C, D}^{m}-M_{a_{j+1} \ldots a_{|A|}} \mathcal{W}_{a_{1} \ldots a_{j}, B, C, D}^{m}\right) \\
& +(A \leftrightarrow B, C, D) \\
Q M_{A, B, C, D}^{m}= & k_{A}^{m} M_{A} M_{B, C, D} \\
& +\sum_{j=1}^{|A|-1}\left(M_{a_{1} \ldots a_{j}} M_{a_{j+1} \ldots a_{|A|}, B, C, D}^{m}-M_{a_{j+1} \ldots a_{|A|}} M_{a_{1} \ldots a_{j}, B, C, D}^{m}\right) \\
& +(A \leftrightarrow B, C, D) .
\end{aligned}
$$


The vectorial building block $M_{A, B, C, D}^{m}$ causes the first explicit appearance of multiparticle vector superfield $A_{B}^{m}$, see (5.19). Its multiparticle equation of motion in (4.6) is required to derive (5.21) at arbitrary multiplicities $|A|, \ldots,|D|$. With $M_{B}=\lambda^{\alpha} \mathcal{A}_{\alpha}^{B}$ and the $W_{B}^{\alpha}, F_{B}^{m n}$ constituents in the definition (5.4) of $M_{A, C, D}$, we have by now seen all the four superfields $\left\{A_{\alpha}^{B}, A_{B}^{m}, W_{B}^{\alpha}, F_{B}^{m n}\right\}$ in the multiparticle vertex operator $U_{B}$ entering one-loop BRST blocks.

\subsection{Vector BRST cohomology at one-loop}

It is interesting to study vectorial uplifts $M_{A} M_{B, C, D} \rightarrow M_{A} M_{B, C, D, i}^{m}$ of the scalar BRST invariants $C_{1 \mid A, B, C}$ as given by (5.13). The deconcatenation terms due to the second line of (5.21) drop out from the BRST variation, but the contributions from the first line remain where the free vector index is carried by external momenta $k^{m}$. The first example

$$
B_{1 \mid 2,3,4,5}^{m} \equiv M_{1} M_{2,3,4,5}^{m}, \quad Q B_{1 \mid 2,3,4,5}^{m}=-\left[k_{2}^{m} E_{12} M_{3,4,5}+(2 \leftrightarrow 3,4,5)\right]
$$

obtained from $C_{1 \mid 2,3,4}$ appeared in the context of the five point closed string amplitude [20]. Its six point generalization

$$
B_{1 \mid 23,4,5,6}^{m} \equiv M_{1} M_{23,4,5,6}^{m}+M_{1} \otimes_{2} B_{2 \mid 3,4,5,6}^{m}-M_{1} \otimes_{3} B_{3 \mid 2,4,5,6}^{m}
$$

resembles $C_{1 \mid 23,4,5}$ and satisfies,

$$
Q B_{1 \mid 23,4,5,6}^{m}=-k_{2}^{m} E_{132} M_{4,5,6}+k_{3}^{m} E_{123} M_{4,5,6}+\left[k_{4}^{m} V_{4} C_{1 \mid 23,5,6}+(4 \leftrightarrow 5,6)\right] .
$$

The higher-multiplicity examples are similarly analysed. The fact that the $k_{i}^{m}$ coefficients in both (5.22) and (5.23) are $Q$-exact ${ }^{16}$ hints the existence of vectorial BRST invariants.

Vector BRST invariants can be constructed using the same procedures as in the scalar case. We assume that each superspace expression $M_{i} M_{A, B, C, D}^{m}$ with single-particle label $i$ admits a BRST-invariant completion of the form

$$
C_{i \mid A, B, C, D}^{m} \equiv M_{i} M_{A, B, C, D}^{m}+\sum_{\{\delta\} \neq \emptyset} M_{i\{\delta\}} f_{\{\delta\}}^{m}\left(k_{.}^{m} M_{,,, \cdot,}, M_{\cdot, \cdot, \cdot, \cdot}^{m}\right) .
$$

Any term in the sum over ordered subsets $\{\delta\}$ of $A \cup B \cup C \cup D$ incorporates label $i$ in a multiparticle $M_{i\{\delta\}}$. The accompanying $f_{\{\delta\}}^{m}$ denote vector combinations of building blocks $M_{E, F, G, H}^{m}($ see $(5.19))$ and $k_{H}^{m} M_{E, F, G}$.

Then, as already argued in the scalar case, $Q^{2}=0$ and the assumed uniqueness of the BRST completions (5.6) and (5.24) implies that the BRST variation (5.21) can be rewritten as

$Q M_{A, B, C, D}^{m}=C_{a_{1} \mid a_{2} \ldots a_{|A|}, B, C, D}^{m}-C_{a_{|A|} \mid a_{1} \ldots a_{|A|-1}, B, C, D}^{m}+\delta_{|A|, 1} k_{a_{1}}^{m} C_{a_{1} \mid B, C, D}+(A \leftrightarrow B, C, D)$.

In the single-particle case $|A|=1$, the first line of (5.21) generates the defining term $M_{i} M_{B, C, D}$ of a scalar invariant (5.6), and the definition $C_{i \mid \emptyset, B, C, D}^{m} \equiv 0$ must then be

\footnotetext{
${ }^{16}$ Recall that $E_{12 \ldots p}=Q M_{12 \ldots p}$ and $V_{4} C_{1 \mid 23,5,6}=Q\left(M_{4} \otimes_{1} C_{1 \mid 23,5,6}\right)$ by Lemma 1.
} 
used to suppress the first two terms of (5.25). We take advantage of (5.25) to rewrite $Q\left(M_{i} M_{A, B, C, D}^{m}\right)$ in terms of $M_{i} C_{j \mid B, C, D, E}^{m}$ and $M_{i} C_{j \mid B, C, D} k_{E}^{m}$. Hence, the BRST completions $f_{\{\delta\}}^{m}$ in (5.24) are determined by the BRST ancestors of $M_{i} C_{j \mid B, C, D}$ and $M_{i} C_{j \mid B, C, D, E}^{m}$. The former are already known from Lemma 1, and the latter can be easily found by the same properties of the concatenation operation (5.9). Similar to the scalars $M_{B, C, D}$, the $\otimes_{j}$ action on vector BRST blocks is defined to be trivial,

$$
M_{i} \otimes_{a_{1}}\left(M_{a_{1} a_{2} \ldots a_{|A|}}^{m} M_{B, C, D, E}\right) \equiv\left(M_{i} \otimes_{a_{1}} M_{a_{1} a_{2} \ldots a_{|A|}}\right) M_{B, C, D, E}^{m} .
$$

Lemma 2. If $C_{j \mid A, B, C, D}^{m}$ as defined by (5.24) is BRST closed, then its concatenation satisfies

$$
Q\left(M_{i} \otimes_{j} C_{j \mid A, B, C, D}^{m}\right)=M_{i} C_{j \mid A, B, C, D}^{m}
$$

Proof. The arguments used in the proof of Lemma 1 can be repeated for vectorial combinations $f_{\{\delta\}}^{m}$ of $k_{\cdot}^{m} M_{,,,,}$and $M_{\cdot, \cdot, \cdot, \cdot}^{m}$ at ghost number two as well as

$$
Q\left(C_{j \mid A, B, C, D}^{m}\right)=\sum_{\{\sigma\}} M_{j\{\sigma\}} F_{\{\sigma\}}^{m}\left(k_{.}^{m} M . M_{\cdot, \cdot, \cdot,}, M_{.} M_{\cdot, \cdot, \cdot, \cdot}^{m}\right)=0 .
$$

The ghost-number-three objects $F_{\{\sigma\}}^{m}$ built from $k_{.}^{m} M_{.} M_{.,,,}$and $M_{.} M_{\cdot, \cdot, \cdot,}^{m}$ again vanish by independence of the $M_{j\{\sigma\}}$ such that

$$
\begin{aligned}
Q\left(M_{i} \otimes_{j} C_{j \mid A, B, C, D}^{m}\right) & =M_{i}\left\{M_{j} M_{A, B, C, D}^{m}+\sum_{\{\delta\} \neq \emptyset} M_{j\{\delta\}} f_{\{\delta\}}^{m}\right\}+\sum_{\{\sigma\}} M_{i j\{\sigma\}} F_{\{\sigma\}}^{m} \\
& =M_{i} C_{j \mid A, B, C, D}^{m}
\end{aligned}
$$

by $(5.24)$.

Then, again in analogy with the scalar case, a recursive definition of vector invariants can be obtained from (5.25) as follows,

$$
\begin{gathered}
C_{i \mid A, B, C, D}^{m} \equiv M_{i} M_{A, B, C, D}^{m}+\left[\delta_{|A|, 1} k_{a_{1}}^{m} M_{i} \otimes_{a_{1}} C_{a_{1} \mid B, C, D}+M_{i} \otimes_{a_{1}} C_{a_{1} \mid a_{2} \ldots a_{|A|}, B, C, D}^{m}\right. \\
\left.-M_{i} \otimes_{a_{|A|}} C_{a_{|A|} \mid a_{1} \ldots a_{|A|-1}, B, C, D}^{m}+(A \leftrightarrow B, C, D)\right] .
\end{gathered}
$$

BRST invariance follows from (5.25) and Lemma 2. In view of the four slots $A, B, C, D$, the bracket $[\ldots]$ on the right hand side of (5.28) contains $8-n$ terms where $n$ is the number of single-particle slots.

The first non-trivial applications of (5.28) are easily checked to be BRST closed,

$$
\begin{aligned}
C_{1 \mid 2,3,4,5}^{m}= & M_{1} M_{2,3,4,5}^{m}+\left[k_{2}^{m} M_{1} \otimes_{2} C_{2 \mid 3,4,5}+(2 \leftrightarrow 3,4,5)\right] \\
= & M_{1} M_{2,3,4,5}^{m}+\left[k_{m}^{2} M_{12} M_{3,4,5}+(2 \leftrightarrow 3,4,5)\right] \\
C_{1 \mid 23,4,5,6}^{m}= & M_{1} M_{23,4,5,6}^{m}+M_{1} \otimes_{2} C_{2 \mid 3,4,5,6}^{m}-M_{1} \otimes_{3} C_{3 \mid 2,4,5,6}^{m} \\
& \quad+\left[k_{4}^{m} M_{1} \otimes_{4} C_{4 \mid 23,5,6}+(4 \leftrightarrow 5,6)\right] \\
= & M_{1} M_{23,4,5,6}^{m}+M_{12} M_{3,4,5,6}^{m}-M_{13} M_{2,4,5,6}^{m} \\
& +\left[k_{3}^{m} M_{123} M_{4,5,6}+(3 \leftrightarrow 4,5,6)\right]-\left[k_{2}^{m} M_{132} M_{4,5,6}+(2 \leftrightarrow 4,5,6)\right] \\
& +\left[k_{4}^{m} M_{14} M_{23,5,6}+M_{142} M_{3,5,6}-M_{143} M_{2,5,6}+(4 \leftrightarrow 5,6)\right],
\end{aligned}
$$

and higher-multiplicity analogues are also straightforward to obtain. Component expansion up to multiplicity seven are available from [46]. 


\section{Conclusion and outlook}

In this work, we have constructed multiparticle vertex operators $U^{12 \ldots p}$ through a recursive prescription described in subsection 3.4. This generalizes and streamlines the earlier construction of BRST-covariant building blocks in [12-14]. The coefficients of conformal weight-one fields $\left\{\partial \theta^{\alpha}, \Pi^{m}, d_{\alpha}, N^{m n}\right\}$ in $U_{B}$ are interpreted as multiparticle superfields $K_{B} \in\left\{A_{\alpha}^{B}, A_{m}^{B}, W_{B}^{\alpha}, F_{m n}^{B}\right\}$ of ten-dimensional SYM with shorthands $B=12 \ldots p$ for external $p$-particle trees. Their equations of motions are shown to have the same structure as their single-particle relatives - see (3.61) versus (2.1). In addition, they are enriched by contact terms where the multiparticle label $B$ is distributed into two smaller subsets.

These multiparticle SYM fields furnish a kinematic analogue of the structure constants $f^{a b c}$ of the color sector, and their Lie symmetries (3.1) guarantee that the tree-level subgraphs described by $K_{B}$ are compatible with the BCJ duality between color and kinematics [26]. Since the BCJ duality has been observed to hold in various dimensions, it will be interesting to explore lower-dimensional setups for multiparticle equations of motion.

It is worth emphasizing that the Lie-algebraic nature of the BRST blocks is completely general and can be understood in terms of its basic SYM superfield constituents. The particular combinations of single-particle superfields constituting their multiparticle generalizations defined in this paper are suggested by OPE computations among vertex operators in the pure spinor formalism. Moreover, they are in lines with the BRST cohomology organization of scattering amplitudes suggested in [45] and brought to fruition in $[10,12-14]$. Given the general Lie symmetries obeyed by the multiparticle SYM superfields and their appearance in the OPEs of vertex operators, it is therefore natural to suspect that the BCJ duality between color and kinematics might be valid at the level of external tree subdiagrams to all loop-orders [63].

In section 5, which is devoted to one-loop applications, the zero mode saturation of the minimal pure spinor formalism [19] singles out some elementary combinations of $K_{B}$ with beneficial BRST properties - such as scalars $M_{A, B, C}$ in (5.4) and vectors $M_{A, B, C, D}^{m}$ in (5.19). We have derived recursions (5.12) and (5.28) to construct scalar and vectorial cohomology elements at arbitrary multiplicity out of $M_{D} M_{A, B, C}$ and $M_{E} M_{A, B, C, D}^{m}$. We can learn from the five-point results in $[20,21]$ that vector invariants are crucial for oneloop amplitudes among closed string states, where cross-contractions between left- and right-moving worldsheet fields occur.

Since the number of left-right contractions is unbounded for multiparticle one-loop amplitudes, the need for BRST invariants extends to tensors of arbitrary rank. The construction of tensorial BRST-blocks generalizing $M_{A, B, C}$ and $M_{A, B, C, D}^{m}$ as well as their BRSTinvariant embedding into full-fledged closed string amplitudes is left for future work [39]. Moreover, it remains to clarify how these tensors are related to the gauge anomaly of open superstring amplitudes and its cancellation [36, 37].

For all of the aforementioned building blocks, the superspace representation in terms of elementary SYM superfields is explicitly accessible from this work. So the zero mode integration prescription of the schematic form $\left\langle\lambda^{3} \theta^{5}\right\rangle=1$ [17] as automated in [32] allows to derive supermultiplet components in terms of gluon polarization vectors and gaugino 
wave functions. The gluon components of all the scalar and vector cohomology elements up to multiplicity seven can be found on the website [46].

Finally, it is worthwhile to note that the (non-minimal) pure spinor formalism can be interpreted as a critical topological string [18]. As shown in [60], the BRST cohomology of a topological CFT is endowed with a Gerstenhaber algebra structure and it would therefore be interesting to investigate possible connections with the BRST covariance property of multiparticle vertex operators. As pointed out by in [59], the associated Gerstenhaber bracket among vertex operators is a promising starting point to relate string amplitudes of different particle content. These references motivate further study of multiparticle vertex operators in view of both mathematical structures and applications to scattering of massive string states.

\section{Acknowledgments}

We thank Tim Adamo for useful comments on the draft. CRM and OS acknowledge financial support by the European Research Council Advanced Grant No. 247252 of Michael Green. OS is grateful to DAMTP for hospitality during various stages of this work.

\section{A Physics of BRST blocks versus mathematics of cubic graphs}

In this appendix we connect the recursive construction of BRST blocks with mathematical operations on planar binary trees, see [3, 4, 24] and references therein. As explained in the references, a mapping between planar binary trees and iterated brackets gives rise to an explicit Lie algebra basis construction. This will be used to manifest the Lie symmetries (3.1) of the BRST blocks and emphasize their connection with cubic graphs which play a central role for the duality between color and kinematics [26].

\section{A.1 Iterated bracket notation}

The antisymmetry of a rank-two BRST block $K_{a_{1} a_{2}}$ can be made manifest with the notation $K_{\left[a_{1}, a_{2}\right]} \equiv K_{a_{1} a_{2}}$. In general, the defining property of a rank- $p$ BRST block to satisfy all Lie symmetries $£_{k}$ with $k \leq p$ motivates the following notation with iterated brackets,

$$
\begin{aligned}
K_{\left[a_{1}, a_{2}\right]} & \equiv K_{a_{1} a_{2}} \\
K_{\left[\left[a_{1}, a_{2}\right], a_{3}\right]} & \equiv K_{\left[a_{1} a_{2}, a_{3}\right]} \equiv K_{a_{1} a_{2} a_{3}} \\
& \vdots \\
K_{\left[\left[\left[\ldots\left[\left[a_{1}, a_{2}\right], a_{3}\right], \ldots\right], a_{p-1}\right], a_{p}\right]} & \equiv K_{\left[a_{1} a_{2} \ldots a_{p-1}, a_{p}\right]} \equiv K_{a_{1} a_{2} \ldots a_{p}} .
\end{aligned}
$$

The virtue of this bracket structure for the duality between color and kinematics was already emphasized in [58]. The above notation reminds of the recursive definition of BRST blocks which features a repeated antisymmetrization $\left(a_{1} a_{2} \ldots a_{j-1} \leftrightarrow a_{j}\right)$ with $j=$ $2,3, \ldots, p$. Moreover, they are in lines with the symmetry matching (3.2) with color factors upon expanding the structure constants

$$
K_{\left[\left[\left[\ldots\left[\left[a_{1}, a_{2}\right], a_{3}\right], \ldots\right], a_{p-1}\right], a_{p}\right]} \leftrightarrow \operatorname{tr}\left(\left[\left[\left[\ldots\left[\left[T^{a_{1}}, T^{a_{2}}\right], T^{a_{3}}\right], \ldots\right], T^{a_{p-1}}\right], T^{a_{p}}\right]\right) .
$$



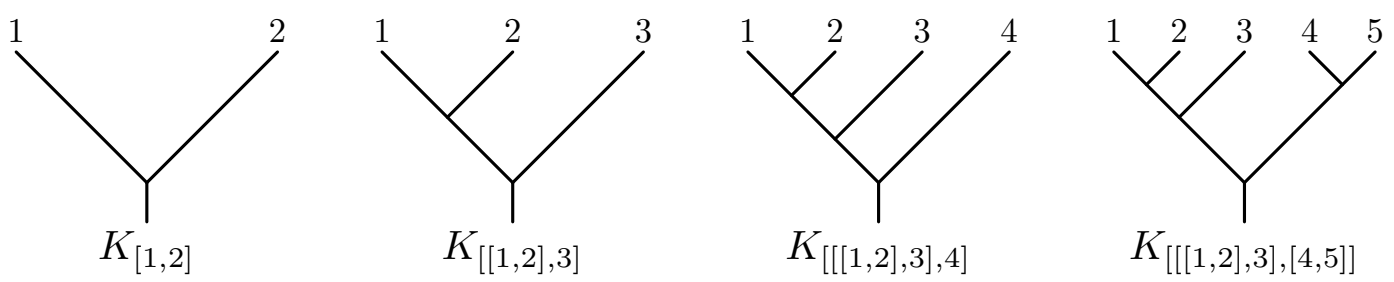

Figure 7. Examples of the mapping between cubic graphs with one leg off-shell and BRST blocks. Together with the conventions (A.1), the fact that the BRST blocks furnish an explicit representation of the "Jacobi identity of trees" of the type discussed in [26] becomes manifest.

Furthermore, more general bracketing patterns can always be brought to the canonical form (A.1) by using the antisymmetry and Jacobi identity satisfied by the brackets. For example,

$$
\begin{aligned}
& K_{[[1,[2,3]], 4]}=-K_{[[[2,3], 1], 4]}=-K_{2314} \\
& K_{[[1,2],[3,4]]}=K_{[[[1,2], 3], 4]}-K_{[[[1,2], 4], 3]}=K_{1234}-K_{1243} .
\end{aligned}
$$

Using the iterated bracket notation introduced above the explicit expressions for the Lie symmetries (3.1) can be easily reproduced. To see this one uses the antisymmetry of the outer commutator to write $K_{[A, B]}=-K_{[B, A]}$ (here $A$ and $B$ represent arbitrary combinations of brackets acting on the multiparticle labels) and applies the conventions (A.1). For example, the $£_{4}$ symmetry in $(3.1)$ is reproduced by $K_{[[1,2],[3,4]]}=-K_{[[3,4],[1,2]]}$, which implies that $K_{1234}-K_{1243}=-K_{3412}+K_{3421}$.

\section{A.2 Diagrammatic representation of BRST blocks and their recursion}

In the mathematics literature, such as $[3,4,24]$ and references therein, there is a well-known mapping between planar binary trees ${ }^{17}$ and iterated brackets which is used to construct an explicit Lie algebra basis [3]. Given the iterated bracket convention discussed above, this can be immediately borrowed to create a mapping between cubic graphs with one leg off-shell and BRST blocks, ${ }^{18}$ see figure 7 . The algorithm is as follows. First index the external legs with the labels $\{1,2, \ldots, n\}$ from left to right and, starting from the left, for each vertex associate the bracket $[A, B]$ where $A$ and $B$ represent the labels to the left and to the right of the vertex (which may already be partially bracketed themselves).

Given the mapping described above, it is interesting to consider the effect of the grafting $[4,24]$ operation of trees in their associated BRST block images. The grafting of two planar binary trees $t_{A}$ and $t_{B}$ is represented by $t_{A} \vee t_{B}$ and joins the roots (i.e. the off-shell leg) of $t_{A}$ and $t_{B}$ to create a new root. It is not difficult to see that if $K_{A}$ and $K_{B}$ are the BRST blocks associated with $t_{A}$ and $t_{B}$ then $t_{A} \vee t_{B}$ is mapped to $K_{[A, B]}$, see figure 8 . Note that the definition of $\hat{A}_{\alpha}^{123 \ldots p}$ in section 3 can be interpreted (up to the redefinitions by $H_{12 \ldots p}$ ) as the grafting of two trees with multiplicity $p-1$ and 1 .

\footnotetext{
${ }^{17}$ The precise definitions can be found in $[3,24]$. But for our purposes, a planar binary tree is nothing more than a cubic graph with one leg off-shell.

${ }^{18}$ This prescription was already hinted (up to an overall sign) in the diagrammatic derivation of the symmetries obeyed by the building block $T_{B}$ discussed in $[13,14]$. The mapping now extends to the whole class of multiparticle superfields $K_{B} \in\left\{A_{\alpha}^{B}, A_{B}^{m}, W_{B}^{\alpha}, F_{B}^{m n}\right\}$.
} 

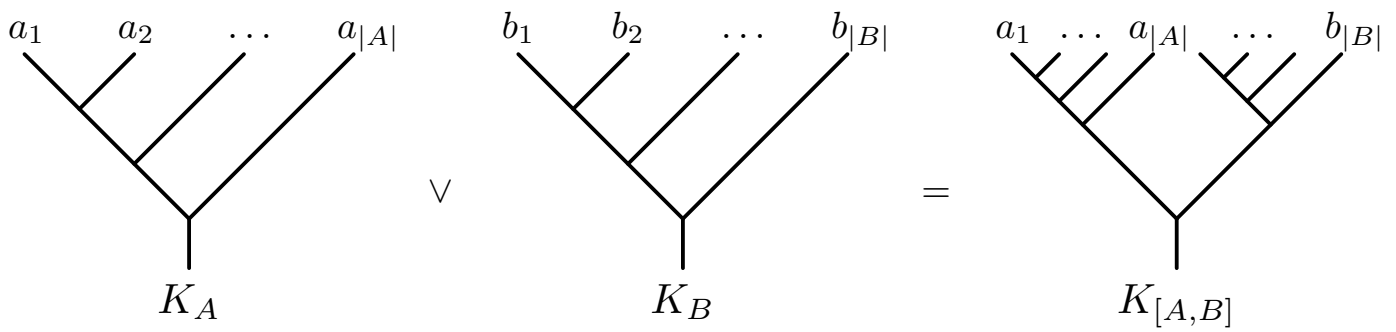

Figure 8. The grafting operation on trees and its corresponding mapping in terms of BRST blocks.

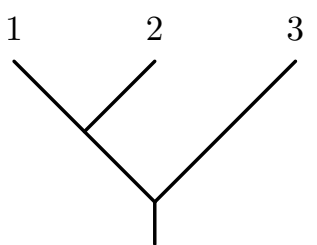

$\frac{K_{[[1,2], 3]}}{s_{12} s_{123}}$

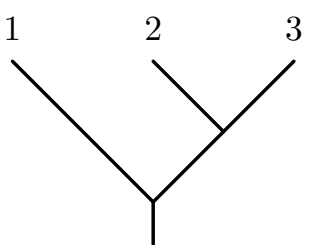

$\frac{K_{[1,[2,3]]}}{s_{23} s_{123}}$

Figure 9. A diagrammatic derivation of the Berends-Giele current $\mathcal{K}_{123}$. The two cubic graphs correspond to the two possibilities of bracketing three external legs, [[12]3], [1[23]] and give rise to the expression $\mathcal{K}_{123}=\frac{K_{123}}{s_{12} s_{123}}+\frac{K_{321}}{s_{23} s_{123}}$ under the mapping described below together with the conventions (A.1).

\section{A.3 Diagrammatic construction of Berends-Giele currents}

It is possible to find the explicit expressions of Berends-Giele currents $\mathcal{K}_{B}$ in terms of BRST blocks $K_{B}$ with a diagrammatic prescription which uses the mapping discussed above. This can be used as an alternative to the inverse momentum kernel formula given in (4.1).

The Berends-Giele current with multiplicity $p$ is obtained by the sum of the expressions associated with all the $p+1$ cubic graphs with one leg off-shell, whose total number is given by the Catalan number $C_{p-1}$. It is convenient to recall that the Catalan number $C_{p-1}$ represents the number of different ways that $p$ factors can be bracketed and each possibility has a direct representation in terms of cubic graphs. To each graph a BRST block $K_{[[\ldots, \ldots], \ldots]}$ is assigned with the corresponding bracketing (which reflects the vertex structure). In addition, an inverse Mandelstam invariant should be multiplied for each non-external edge.

The two possibilities of bracketing three external legs, namely [[12]3] and [1[23]], give rise to the expression for $\mathcal{K}_{123}$ under the mapping described above, see figure 9 . Similarly, the five different bracketing possibilities of four external legs

$$
[[[12] 3] 4],[[1[23]] 4],[[12][34]],[1[2[34]]],[1[[23] 4]]
$$

and their corresponding mapping in terms of cubic graphs and BRST blocks leading to the expression $\mathcal{K}_{1234}$ were depicted in figure 6. Higher-multiplicity examples are similarly handled. 


\section{A.4 Different superfield representations versus Lie symmetries}

The definition of the hatted BRST blocks at multiplicity $p$ has an explicit antisymmetrization of the form $12 \ldots p-1 \leftrightarrow p$, where $p$ is a single-particle label. As discussed above, the resulting BRST block is represented by a iterated bracket where the second slot of the outer bracket is a single-particle label. This motivates to check the outcome of a more general hatted superfield definition featuring a multiparticle label instead of $p$. As the brief discussion below suggests, the result is compatible with a linear combination of the "standard" BRST blocks following from the iterated bracket notation.

To see this, consider a rank-four hatted BRST block with the symmetry structure $(12 \leftrightarrow 34)$ instead of $(123 \leftrightarrow 4)$ as in $(3.35)$. For example,

$$
\hat{A}_{\alpha}^{[1,2],[3,4]]}=-\frac{1}{2}\left[A_{\alpha}^{12}\left(k^{12} \cdot A^{34}\right)+A_{m}^{12}\left(\gamma^{m} W^{34}\right)_{\alpha}-(12 \leftrightarrow 34)\right] .
$$

It is not difficult to show that $\hat{V}_{[[1,2],[3,4]]} \equiv \lambda^{\alpha} \hat{A}_{\alpha}^{[[1,2],[3,4]]}$ satisfies

$$
Q \hat{V}_{[[1,2],[3,4]]}=\left(k^{1} \cdot k^{2}\right)\left[V_{2} \hat{V}_{341}-(1 \leftrightarrow 2)\right]+\left(k^{3} \cdot k^{4}\right)\left[V_{3} \hat{V}_{124}-(3 \leftrightarrow 4)\right]+\left(k^{12} \cdot k^{34}\right) V_{12} V_{34} .
$$

where the equation of motion for $D_{(\alpha} \hat{A}_{\beta)}$ was contracted with $\lambda^{\alpha} \lambda^{\beta}$ for the sake of simplicity. Therefore the redefinition

$$
V_{[[1,2],[3,4]]} \equiv \hat{V}_{[[1,2],[3,4]]}+\left(k^{1} \cdot k^{2}\right)\left[V_{2} H_{341}-(1 \leftrightarrow 2)\right]+\left(k^{3} \cdot k^{4}\right)\left[V_{3} H_{124}-(3 \leftrightarrow 4)\right]
$$

satisfies

$$
Q V_{[[1,2],[3,4]]}=Q V_{1234}-Q V_{1243} .
$$

This is compatible with the expectation from the bracket notation since $V_{[[1,2],[3,4]]}=V_{1234}-$ $V_{1243}$, see (A.3).

\section{B BCJ relations and one-loop scalar cohomology elements}

The scalar cohomology elements $C_{1 \mid A, B, C}$ constructed in section 5.3 were argued in [10] to be linear combinations of SYM tree-level amplitudes multiplied by quadratic polynomials of Mandelstam invariants. Momentum conservation as well as BCJ and KK relations among color ordered SYM amplitudes $A^{\mathrm{YM}}(\ldots)[26,30,31]$ lead to a multitude of different such representations for $C_{1 \mid A, B, C}$. In the following, we provide convenient representations at all multiplicities ${ }^{19}$ in the sense that the total number of terms is systematically reduced and inverse powers of Mandelstam invariants are avoided. As we shall see, these $A^{\mathrm{YM}}$ representations of $C_{1 \mid A, B, C}$ are intriguingly related to BCJ relations among tree-level amplitudes.

\footnotetext{
${ }^{19}$ The explicit representation given at multiplicity five in [10] fails to satisfy the above criterion of having local Mandelstam coefficients along with $A^{\mathrm{YM}}(\ldots)$. The six-point representation was given only indirectly as an expansion in terms of $A^{F^{4}}$, which represent the $\alpha^{\prime 2}$ corrections of the string tree-level amplitudes.
} 


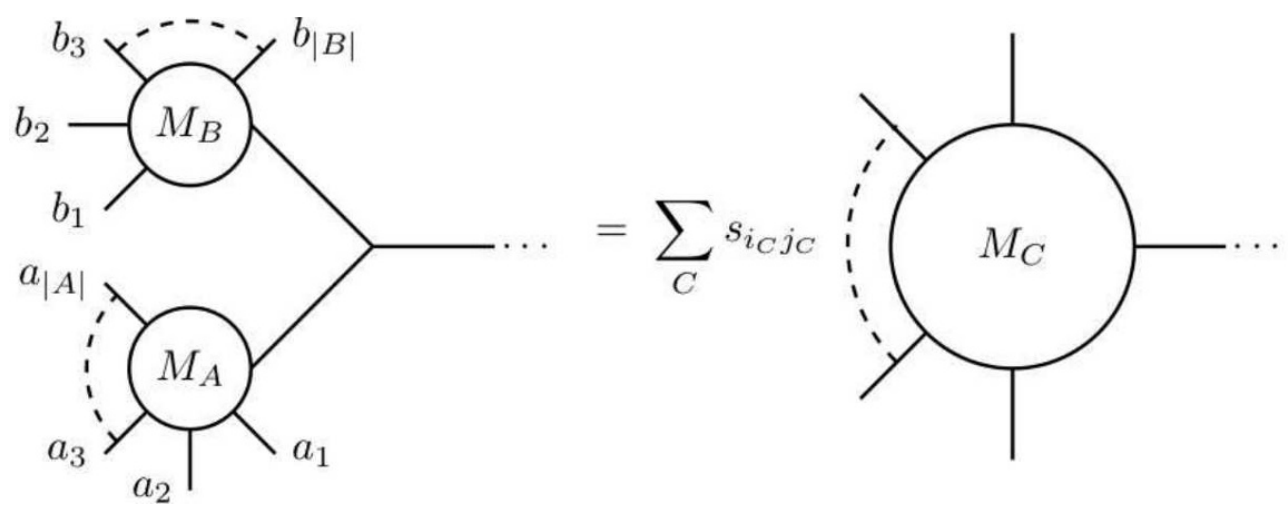

Figure 10. Diagrammatic interpretation of $M_{S[A, B]}$.

\section{B.1 A shuffle formula for BCJ relations}

Let us first define an operation $S[A, B]$ which concatenates two multiparticle labels $A$ and $B$ with Berends-Giele symmetries (see section 4.1) into one such set,

$$
M_{S[A, B]} \equiv \sum_{i=1}^{|A|} \sum_{j=1}^{|B|}(-1)^{i-j+|A|-1} s_{a_{i} b_{j}} M_{\left(a_{1} a_{2} \ldots a_{i-1} ш a_{|A|} a_{|A|-1} \ldots a_{i+1}\right) a_{i} b_{j}\left(b_{j-1} \ldots b_{2} b_{1} ш b_{j+1} \ldots b_{|B|}\right)} .
$$

One can interpret $M_{S[A, B]}$ in (B.1) as attaching two Berends-Giele currents $M_{A}$ and $M_{B}$ to a cubic vertex and expressing the resulting diagram in terms of $M_{C}$ at overall multiplicity $|C|=|A|+|B|$, see figure 10. For example,

$$
\begin{aligned}
M_{S[1,2]} & =s_{12} M_{12} \\
M_{S[1,23]} & =s_{12} M_{123}-s_{13} M_{132} \\
M_{S[1,234]} & =s_{12} M_{1234}-s_{13}\left(M_{1324}+M_{1342}\right)+s_{14} M_{1432} \\
M_{S[12,34]} & =-s_{13} M_{2134}+s_{14} M_{2143}+s_{23} M_{1234}-s_{24} M_{1243} .
\end{aligned}
$$

It turns out that the $S[A, B]$ product defined by (B.1) can be used to generate BCJ relations among tree amplitudes [26]. Recalling [12] that SYM tree amplitudes are given by $A^{\mathrm{YM}}(1,2, \ldots, n)=\left\langle V_{1} E_{23 \ldots n}\right\rangle$, BCJ relations among $A^{\mathrm{YM}}$ can be written as

$$
\left\langle V_{1} E_{S[A, B]}\right\rangle=0, \quad \forall A, B,
$$

for example

$$
\begin{aligned}
0=\left\langle V_{1} E_{S[2,34]}\right\rangle= & s_{23} A^{\mathrm{YM}}(1,2,3,4)-s_{24} A^{\mathrm{YM}}(1,2,4,3) \\
0=\left\langle V_{1} E_{S[2,345]}\right\rangle= & s_{23} A^{\mathrm{YM}}(1,2,3,4,5)+s_{25} A^{\mathrm{YM}}(1,2,5,4,3) \\
& -s_{24}\left(A^{\mathrm{YM}}(1,2,4,3,5)+A^{\mathrm{YM}}(1,2,4,5,3)\right) \\
0=\left\langle V_{1} E_{S[23,45]}\right\rangle= & s_{34} A^{\mathrm{YM}}(1,2,3,4,5)-s_{35} A^{\mathrm{YM}}(1,2,3,5,4) \\
& -s_{24} A^{\mathrm{YM}}(1,3,2,4,5)+s_{25} A^{\mathrm{YM}}(1,3,2,5,4) . \\
0=\left\langle V_{1} E_{S[2,3456]}\right\rangle= & s_{23} A^{\mathrm{YM}}(1,2,3,4,5,6)-s_{26} A^{\mathrm{YM}}(1,2,6,5,4,3) \\
& -s_{24}\left(A^{\mathrm{YM}}(1,2,4,3,5,6)+A^{\mathrm{YM}}(1,2,4,5,3,6)+A^{\mathrm{YM}}(1,2,4,5,6,3)\right) \\
& +s_{25}\left(A^{\mathrm{YM}}(1,2,5,6,4,3)+A^{\mathrm{YM}}(1,2,5,4,6,3)+A^{\mathrm{YM}}(1,2,5,4,3,6)\right) . \\
&
\end{aligned}
$$


Similar formulæ for BCJ relations using shuffle products can be found in $^{20}[7,8,49]$. We have explicitly verified that (B.3) holds up to multiplicity $|A|+|B|+1=7$ using the data from [46].

\section{B.2 $\left\langle C_{1 \mid A, B, C}\right\rangle$ from the BCJ shuffle formula}

Since (B.3) also holds for $A$ or $B$ of the form $S[C, D]$, we can iterate the product (B.1) and generate further vanishing identities for SYM subamplitudes from $E_{S[S[A, B], C]}$. Any partition of $A, B$ and $C$ leads to an $A^{\mathrm{YM}}$ relation with local polynomials of degree two in Mandelstam invariants. The examples

$$
\begin{aligned}
0=\left\langle V_{1} E_{S[S[2,3], 4]}\right\rangle=s_{23} s_{34} & A^{\mathrm{YM}}(1,2,3,4)-s_{23} s_{24} A^{\mathrm{YM}}(1,3,2,4) \\
0=\left\langle V_{1} E_{S[S[4,5], 23]}\right\rangle=-s_{34} & s_{45} A^{\mathrm{YM}}(1,2,3,4,5)+s_{35} s_{45} A^{\mathrm{YM}}(1,2,3,5,4) \\
& +s_{24} s_{45} A^{\mathrm{YM}}(1,3,2,4,5)-s_{25} s_{45} A^{\mathrm{YM}}(1,3,2,5,4) \\
0=\left\langle V_{1} E_{S[S[5,6], 234]}\right\rangle=-s_{56} & s_{45} A^{\mathrm{YM}}(1,2,3,4,5,6)+s_{56} s_{46} A^{\mathrm{YM}}(1,2,3,4,6,5) \\
& +s_{56} s_{35} A^{\mathrm{YM}}(1,2,4,3,5,6)-s_{56} s_{36} A^{\mathrm{YM}}(1,2,4,3,6,5) \\
& +s_{56} s_{35} A^{\mathrm{YM}}(1,4,2,3,5,6)-s_{56} s_{36} A^{\mathrm{YM}}(1,4,2,3,6,5) \\
& -s_{56} s_{25} A^{\mathrm{YM}}(1,4,3,2,5,6)+s_{56} s_{26} A^{\mathrm{YM}}(1,4,3,2,6,5) \\
0=\left\langle V_{1} E_{S[S[6,45], 23]}\right\rangle=+s_{46} s_{34} A^{\mathrm{YM}}(1,2,3,4,5,6)+s_{56} s_{34} A^{\mathrm{YM}}(1,2,3,4,5,6) & +s_{46} s_{34} A^{\mathrm{YM}}(1,2,3,4,6,5)-s_{46} s_{35} A^{\mathrm{YM}}(1,2,3,5,4,6) \\
& -s_{56} s_{35} A^{\mathrm{YM}}(1,2,3,5,4,6)-s_{56} s_{35} A^{\mathrm{YM}}(1,2,3,5,6,4) \\
& -s_{46} s_{36} A^{\mathrm{YM}}(1,2,3,6,4,5)+s_{56} s_{36} A^{\mathrm{YM}}(1,2,3,6,5,4) \\
& -s_{46} s_{24} A^{\mathrm{YM}}(1,3,2,4,5,6)-s_{56} s_{24} A^{\mathrm{YM}}(1,3,2,4,5,6) \\
& -s_{46} s_{24} A^{\mathrm{YM}}(1,3,2,4,6,5)+s_{46} s_{25} A^{\mathrm{YM}}(1,3,2,5,4,6) \\
& +s_{56} s_{25} A^{\mathrm{YM}}(1,3,2,5,4,6)+s_{56} s_{25} A^{\mathrm{YM}}(1,3,2,5,6,4) \\
& +s_{46} s_{26} A^{\mathrm{YM}}(1,3,2,6,4,5)-s_{56} s_{26} A^{\mathrm{YM}}(1,3,2,6,5,4)
\end{aligned}
$$

can be checked to be a consequence of the BCJ relations [26]. Note that $E_{S[S[A, B], C]}$ in the five-point example is chosen as $(A, B, C=4,5,23)$ rather than $(A, B, C=23,4,5)$ in order to minimize the number of terms.

The motivation to delve on the redundant BCJ relations (B.5) in addition to (B.4) stems from their intriguing connection with the $A^{\mathrm{YM}}$ representation of the scalar cohomology elements $C_{1 \mid A, B, C}$. Up to six-points, we have

$$
\begin{aligned}
-\left\langle C_{1 \mid 2,3,4}\right\rangle= & -s_{24} s_{23} A^{\mathrm{YM}}(1,3,2,4) \\
-\left\langle C_{1 \mid 23,4,5}\right\rangle= & -s_{45} s_{34} A^{\mathrm{YM}}(1,2,3,4,5)+s_{45} s_{24} A^{\mathrm{YM}}(1,3,2,4,5) \\
-\left\langle C_{1 \mid 234,5,6}\right\rangle= & -s_{56} s_{45} A^{\mathrm{YM}}(1,2,3,4,5,6)+s_{56} s_{35} A^{\mathrm{YM}}(1,2,4,3,5,6) \\
& +s_{56} s_{35} A^{\mathrm{YM}}(1,4,2,3,5,6)-s_{56} s_{25} A^{\mathrm{YM}}(1,4,3,2,5,6) \\
-\left\langle C_{1 \mid 23,45,6}\right\rangle= & -s_{46} s_{36} A^{\mathrm{YM}}(1,2,3,6,4,5)+s_{56} s_{36} A^{\mathrm{YM}}(1,2,3,6,5,4) \\
& +s_{46} s_{26} A^{\mathrm{YM}}(1,3,2,6,4,5)-s_{56} s_{26} A^{\mathrm{YM}}(1,3,2,6,5,4),
\end{aligned}
$$

${ }^{20}$ We thank Henrik Johansson for pointing out reference [49]. 
and we observe that the expressions on the right hand side can be found by systematically deleting subsets of the terms in (B.5): only those terms in $\left\langle V_{1} E_{S[S[A, B], C]}\right\rangle$ are kept where the Mandelstam bilinear takes the form $s_{a b} s_{a c}$ with $a \in A, b \in B$ and $c \in C$. The following algorithm allows to translate any $\left\langle C_{1 \mid A, B, C}\right\rangle$ into SYM trees:

1. Reorder the labels $A, B$ and $C$ such that $|A| \leq|B| \leq|C|$.

2. Apply the formula (B.1) recursively to evaluate $E_{S[S[A, B], C]}$.

3. Substitute $E_{\sigma_{2} \ldots \sigma_{n}} \rightarrow A^{\mathrm{YM}}\left(1, \sigma_{2}, \ldots, \sigma_{n}\right)$.

4. Keep only the terms containing Mandelstams with labels distributed as in $s_{a b} s_{a c}$, with single-particle labels $a \in A, b \in B$ and $c \in C$. Delete terms of the form $s_{a b} s_{b c}$.

5. The result is $-\left\langle C_{1 \mid A, B, C}\right\rangle$.

We have explicitly checked with the data available from [46] that the algorithm above is correct for all scalar cohomology elements up to multiplicity $|A|+|B|+|C|+1=7$. For example, it leads to

$$
\begin{aligned}
-\left\langle C_{1 \mid 2345,6,7}\right\rangle= & -s_{67} s_{56} A^{\mathrm{YM}}(1,2,3,4,5,6,7)+s_{67} s_{46} A^{\mathrm{YM}}(1,2,3,5,4,6,7) \\
& +s_{67} s_{46} A^{\mathrm{YM}}(1,2,5,3,4,6,7)-s_{67} s_{36} A^{\mathrm{YM}}(1,2,5,4,3,6,7) \\
& +s_{67} s_{46} A^{\mathrm{YM}}(1,5,2,3,4,6,7)-s_{67} s_{36} A^{\mathrm{YM}}(1,5,2,4,3,6,7) \\
& -s_{67} s_{36} A^{\mathrm{YM}}(1,5,4,2,3,6,7)+s_{67} s_{26} A^{\mathrm{YM}}(1,5,4,3,2,6,7) \\
-\left\langle C_{1 \mid 234,56,7}\right\rangle= & -s_{57} s_{47} A^{\mathrm{YM}}(1,2,3,4,7,5,6)+s_{67} s_{47} A^{\mathrm{YM}}(1,2,3,4,7,6,5) \\
& +s_{57} s_{37} A^{\mathrm{YM}}(1,2,4,3,7,5,6)-s_{67} s_{37} A^{\mathrm{YM}}(1,2,4,3,7,6,5) \\
& +s_{57} s_{37} A^{\mathrm{YM}}(1,4,2,3,7,5,6)-s_{67} s_{37} A^{\mathrm{YM}}(1,4,2,3,7,6,5) \\
& -s_{57} s_{27} A^{\mathrm{YM}}(1,4,3,2,7,5,6)+s_{67} s_{27} A^{\mathrm{YM}}(1,4,3,2,7,6,5)
\end{aligned}
$$

and a slightly longer 32 -term representation of $\left\langle C_{1 \mid 23,45,67}\right\rangle$ which is commented out in the $\mathrm{T}_{\mathrm{E} X} \mathrm{X}$ source.

It will be interesting to understand the origin of the intriguing patterns described in this appendix. They hint a deeper connection between the fusion of Berends-Giele currents via (B.1) (see figure 10 for a diagrammatic interpretation), general BCJ relations [26, 49] and the scalar cohomology elements $\left\langle C_{1 \mid A, B, C}\right\rangle$ generating the non-anomalous kinematics in one-loop amplitudes of the open superstring [10].

\section{The explicit expression for $H_{1234}$}

The Lie symmetry of rank-four BRST blocks is restored by the redefinition (3.46) with the following expression for $H_{1234}$ :

$$
4 H_{1234}=H_{1234}^{(a)}-H_{1243}^{(a)}+H_{3412}^{(a)}-H_{3421}^{(a)}
$$


By construction, it is the $£_{4}$ image of a more elementary expression

$$
\begin{aligned}
H_{1234}^{(a)}= & \frac{1}{4}\left(A^{12} \cdot A^{34}\right)+\frac{1}{6}\left(A^{12} \cdot A^{3}\right)\left(k^{3} \cdot A^{4}\right)-\frac{1}{3}\left(A^{12} \cdot A^{3}\right)\left(k^{12} \cdot A^{4}\right)+\frac{1}{2}\left(A^{123} \cdot A^{4}\right) \\
& +\frac{1}{2} A_{m}^{12} A_{n}^{3} F_{m n}^{4}+\frac{1}{6}\left(A^{1} \cdot A^{23}\right)\left(k^{123} \cdot A^{4}\right)-\frac{1}{6}\left(A^{2} \cdot A^{13}\right)\left(k^{123} \cdot A^{4}\right) \\
& +\frac{1}{4}\left(H_{1234}^{(b)}+H_{3412}^{(b)}+H_{1423}^{(b)}+H_{2314}^{(b)}+H_{3124}^{(b)}+H_{2431}^{(b)}\right)
\end{aligned}
$$

with

$$
\begin{aligned}
H_{1234}^{(b)}= & \frac{1}{6}\left(A^{1} \cdot A^{2}\right)\left(k^{4} \cdot A^{3}\right)\left(\left(k^{1} \cdot A^{4}\right)-\left(k^{2} \cdot A^{4}\right)\right) \\
& -\frac{1}{6}\left(A^{1} \cdot A^{2}\right)\left(k^{3} \cdot A^{4}\right)\left(\left(k^{1} \cdot A^{3}\right)-\left(k^{2} \cdot A^{3}\right)\right) \\
& +\frac{1}{3}\left(A^{1} \cdot A^{2}\right)\left[\left(k^{2} \cdot A^{3}\right)\left(k^{1} \cdot A^{4}\right)-\left(k^{1} \cdot A^{3}\right)\left(k^{2} \cdot A^{4}\right)\right] .
\end{aligned}
$$

Open Access. This article is distributed under the terms of the Creative Commons Attribution License (CC-BY 4.0), which permits any use, distribution and reproduction in any medium, provided the original author(s) and source are credited.

\section{References}

[1] N. Berkovits, Infinite tension limit of the pure spinor superstring, JHEP 03 (2014) 017 [arXiv:1311.4156] [INSPIRE].

[2] H. Gomez and E.Y. Yuan, N-point tree-level scattering amplitude in the new Berkovits ${ }^{6}$ string, JHEP 04 (2014) 046 [arXiv: 1312.5485] [INSPIRE].

[3] G. Melançon and C. Reutenauer, Free Lie superalgebras, trees and chains of partitions, J. Alg. Comb. 5 (1996) 337.

[4] J.L. Loday and M.O. Ronco, Hopf algebra of the planar binary trees, Adv. Math. 139 (1998) 293.

[5] L.A. Barreiro and R. Medina, RNS derivation of $N$-point disk amplitudes from the revisited S-matrix approach, arXiv:1310.5942 [INSPIRE].

[6] H. Kawai, D.C. Lewellen and S.H.H. Tye, A relation between tree amplitudes of closed and open strings, Nucl. Phys. B 269 (1986) 1 [INSPIRE].

[7] N.E.J. Bjerrum-Bohr, P.H. Damgaard and P. Vanhove, Minimal basis for gauge theory amplitudes, Phys. Rev. Lett. 103 (2009) 161602 [arXiv:0907.1425] [INSPIRE].

[8] S. Stieberger, Open $\&$ closed vs. pure open string disk amplitudes, arXiv:0907.2211 [INSPIRE].

[9] F.A. Berends and W.T. Giele, Recursive calculations for processes with $n$ gluons, Nucl. Phys. B 306 (1988) 759 [inSPIRE].

[10] C.R. Mafra and O. Schlotterer, The structure of n-point one-loop open superstring amplitudes, arXiv:1203.6215 [INSPIRE].

[11] C.R. Mafra, Simplifying the tree-level superstring massless five-point amplitude, JHEP 01 (2010) 007 [arXiv:0909.5206] [INSPIRE]. 
[12] C.R. Mafra, O. Schlotterer, S. Stieberger and D. Tsimpis, A recursive method for SYM n-point tree amplitudes, Phys. Rev. D 83 (2011) 126012 [arXiv:1012.3981] [INSPIRE].

[13] C.R. Mafra, O. Schlotterer and S. Stieberger, Complete n-point superstring disk amplitude I. pure spinor computation, Nucl. Phys. B 873 (2013) 419 [arXiv:1106.2645] [INSPIRE].

[14] C.R. Mafra, O. Schlotterer and S. Stieberger, Complete n-point superstring disk amplitude II. Amplitude and hypergeometric function structure, Nucl. Phys. B 873 (2013) 461 [arXiv: 1106.2646] [INSPIRE].

[15] E. Witten, Twistor-like transform in ten-dimensions, Nucl. Phys. B 266 (1986) 245 [INSPIRE].

[16] W. Siegel, Superfields in higher dimensional space-time, Phys. Lett. B 80 (1979) 220 [INSPIRE].

[17] N. Berkovits, Super Poincaré covariant quantization of the superstring, JHEP 04 (2000) 018 [hep-th/0001035] [INSPIRE].

[18] N. Berkovits, Pure spinor formalism as an $N=2$ topological string, JHEP 10 (2005) 089 [hep-th/0509120] [INSPIRE].

[19] N. Berkovits, Multiloop amplitudes and vanishing theorems using the pure spinor formalism for the superstring, JHEP 09 (2004) 047 [hep-th/0406055] [INSPIRE].

[20] M.B. Green, C.R. Mafra and O. Schlotterer, Multiparticle one-loop amplitudes and S-duality in closed superstring theory, JHEP 10 (2013) 188 [arXiv:1307.3534] [INSPIRE].

[21] S. He, C.R. Mafra and O. Schlotterer, work in progress.

[22] N. Berkovits and C.R. Mafra, Some superstring amplitude computations with the non-minimal pure spinor formalism, JHEP 11 (2006) 079 [hep-th/0607187] [INSPIRE].

[23] C.R. Mafra, O. Schlotterer and S. Stieberger, Explicit BCJ numerators from pure spinors, JHEP 07 (2011) 092 [arXiv:1104.5224] [INSPIRE].

[24] J-L. Loday and B. Vallette, Algebraic operads, Springer, Heidelberg Germany (2012).

[25] J. Polchinski, String theory. Volume 1: an introduction to the bosonic string, Cambridge University Press, Cambridge U.K. (1998).

[26] Z. Bern, J.J.M. Carrasco and H. Johansson, New relations for gauge-theory amplitudes, Phys. Rev. D 78 (2008) 085011 [arXiv:0805.3993] [InSPIRE].

[27] Z. Bern, L.J. Dixon, M. Perelstein and J.S. Rozowsky, Multileg one loop gravity amplitudes from gauge theory, Nucl. Phys. B 546 (1999) 423 [hep-th/9811140] [INSPIRE].

[28] N.E.J. Bjerrum-Bohr, P.H. Damgaard, T. Sondergaard and P. Vanhove, The momentum kernel of gauge and gravity theories, JHEP 01 (2011) 001 [arXiv:1010.3933] [INSPIRE].

[29] J. Broedel, O. Schlotterer and S. Stieberger, Polylogarithms, multiple zeta values and superstring amplitudes, Fortsch. Phys. 61 (2013) 812 [arXiv:1304.7267] [INSPIRE].

[30] R. Kleiss and H. Kuijf, Multi-gluon cross-sections and five jet production at hadron colliders, Nucl. Phys. B 312 (1989) 616 [inSPIRE].

[31] V. Del Duca, L.J. Dixon and F. Maltoni, New color decompositions for gauge amplitudes at tree and loop level, Nucl. Phys. B 571 (2000) 51 [hep-ph/9910563] [INSPIRE].

[32] C.R. Mafra, PSS: a FORM program to evaluate Pure Spinor Superspace expressions, arXiv: 1007.4999 [INSPIRE]. 
[33] J.A.M. Vermaseren, New features of FORM, math-ph/0010025 [INSPIRE].

[34] M. Tentyukov and J.A.M. Vermaseren, The multithreaded version of FORM, Comput. Phys. Commun. 181 (2010) 1419 [hep-ph/0702279] [INSPIRE].

[35] J.M. Figueroa-O'Farrill, $N=2$ structures in all string theories, J. Math. Phys. 38 (1997) 5559 [hep-th/9507145] [INSPIRE].

[36] M.B. Green and J.H. Schwarz, Anomaly cancellation in supersymmetric D = 10 gauge theory and superstring theory, Phys. Lett. B 149 (1984) 117 [INSPIRE].

[37] M.B. Green and J.H. Schwarz, The hexagon gauge anomaly in type I superstring theory, Nucl. Phys. B 255 (1985) 93 [inSPIRE].

[38] M.A.A. van Leeuwen, A.M. Cohen and B. Lisser, LiE, a package for Lie group computations, Computer Algebra Nederland, Amsterdam The Netherlands (1992).

[39] C.R. Mafra and O. Schlotterer, work in progress.

[40] R. Monteiro and D. O'Connell, The kinematic algebra from the self-dual sector, JHEP 07 (2011) 007 [arXiv:1105.2565] [INSPIRE].

[41] H. Gomez and C.R. Mafra, The closed-string 3-loop amplitude and S-duality, JHEP 10 (2013) 217 [arXiv:1308.6567] [INSPIRE].

[42] P.S. Howe, Pure spinors lines in superspace and ten-dimensional supersymmetric theories, Phys. Lett. B 258 (1991) 141 [Addendum ibid. B 259 (1991) 511] [INSPIRE].

[43] P.S. Howe, Pure spinors, function superspaces and supergravity theories in ten-dimensions and eleven-dimensions, Phys. Lett. B 273 (1991) 90 [INSPIRE].

[44] B.E.W. Nilsson, Pure spinors as auxiliary fields in the ten-dimensional supersymmetric Yang-Mills theory, Class. Quant. Grav. 3 (1986) L41 [InSPIRE].

[45] C.R. Mafra, Towards field theory amplitudes from the cohomology of pure spinor superspace, JHEP 11 (2010) 096 [arXiv: 1007.3639] [INSPIRE].

[46] C.R. Mafra and O. Schlotterer, http://www.damtp.cam.ac.uk/user/crm66/SYM/pss.html.

[47] L. Mason and D. Skinner, Ambitwistor strings and the scattering equations, JHEP 07 (2014) 048 [arXiv:1311.2564] [INSPIRE].

[48] T. Adamo, E. Casali and D. Skinner, Ambitwistor strings and the scattering equations at one loop, JHEP 04 (2014) 104 [arXiv: 1312.3828] [INSPIRE].

[49] Y.-X. Chen, Y.-J. Du and B. Feng, A proof of the explicit minimal-basis expansion of tree amplitudes in gauge field theory, JHEP 02 (2011) 112 [arXiv:1101.0009] [INSPIRE].

[50] M.B. Green and J.H. Schwarz, Covariant description of superstrings, Phys. Lett. B 136 (1984) 367 [inSPIRE].

[51] M.B. Green and J.H. Schwarz, Supersymmetrical string theories, Phys. Lett. B 109 (1982) 444 [inSPIRE].

[52] P. Ramond, Dual theory for free fermions, Phys. Rev. D 3 (1971) 2415 [inSPIRE].

[53] A. Neveu and J.H. Schwarz, Factorizable dual model of pions, Nucl. Phys. B 31 (1971) 86 [INSPIRE].

[54] A. Neveu and J.H. Schwarz, Quark model of dual pions, Phys. Rev. D 4 (1971) 1109 [INSPIRE]. 
[55] N. Berkovits, New higher-derivative $R^{4}$ theorems, Phys. Rev. Lett. 98 (2007) 211601 [hep-th/0609006] [INSPIRE].

[56] A. Ochirov and P. Tourkine, BCJ duality and double copy in the closed string sector, JHEP 05 (2014) 136 [arXiv:1312.1326] [INSPIRE].

[57] C.R. Mafra, O. Schlotterer, S. Stieberger and D. Tsimpis, Six open string disk amplitude in pure spinor superspace, Nucl. Phys. B 846 (2011) 359 [arXiv: 1011. 0994] [InSPIRE].

[58] Z. Bern and T. Dennen, A color dual form for gauge-theory amplitudes, Phys. Rev. Lett. 107 (2011) 081601 [arXiv:1103.0312] [INSPIRE].

[59] G.W. Moore, Symmetries of the bosonic string S matrix, hep-th/9310026 [INSPIRE].

[60] B.H. Lian and G.J. Zuckerman, New perspectives on the BRST algebraic structure of string theory, Commun. Math. Phys. 154 (1993) 613 [hep-th/9211072] [INSPIRE].

[61] M.B. Green, J.H. Schwarz and L. Brink, $N=4$ Yang-Mills and $N=8$ supergravity as limits of string theories, Nucl. Phys. B 198 (1982) 474 [INSPIRE].

[62] W. Siegel, Classical superstring mechanics, Nucl. Phys. B 263 (1986) 93 [InSPIRE].

[63] Z. Bern, J.J.M. Carrasco and H. Johansson, Perturbative quantum gravity as a double copy of gauge theory, Phys. Rev. Lett. 105 (2010) 061602 [arXiv:1004.0476] [InSPIRE].

[64] R. Monteiro and D. O'Connell, The kinematic algebras from the scattering equations, JHEP 03 (2014) 110 [arXiv:1311.1151] [INSPIRE]. 